\title{
INFLUENCE OF BOUNDARY CONDITIONS ON PATTERN FORMATION AND SPATIAL CHAOS IN LATTICE SYSTEMS*
}

\author{
CHIH-WEN SHIH ${ }^{\dagger}$
}

\begin{abstract}
This work elucidates the spatial structure of lattice dynamical systems, which is represented by equilibria of the systems. On a finite lattice, various boundary conditions are imposed. The effect from these boundary conditions on formation of pattern as well as spatial complexity, as the lattice size tends to infinity, is investigated. Two general propositions are proposed as a criteria to demonstrate that this effect is negligible. To illustrate the effectiveness of these criteria, the mosaic patterns in a cellular neural network model on one- and two-dimensional lattices are also studied. On a one-dimensional lattice, the influence of boundary conditions on pattern formation and spatial chaos for mosaic patterns is negligible. This result is justified by verifying the above-mentioned criteria and by using the transition matrices. These appropriately formulated matrices generate all the mosaic patterns on a one-dimensional infinite lattice and on any one-dimensional finite lattice with boundary conditions. On a two-dimensional lattice, two illustrative examples demonstrate that the boundary effect can be dominant. The results and analysis in this investigation have significant implications for circuit design in cellular neural networks.
\end{abstract}

Key words. lattice dynamical system, pattern formation, spatial chaos, cellular neural network

AMS subject classifications. 34C $35,94 \mathrm{C} 99$

PII. S0036139998340650

1. Introduction. The dynamics of spatially extended systems have received considerable attention in recent years [1], [3], [4], [19]. These systems are modeled as partial differential equations or lattice dynamical systems. In the lattice models, while considering the problems on an infinite lattice is more convenient, a more practical consideration would be those problems on a large but finite domain. For a system on a finite lattice, boundary conditions must be imposed and realized. In the investigation of spatial complexity for the lattice systems, it is interesting to see the effect from various boundary conditions. Correspondingly, the following question arises: does the temporal-spatial structure on an infinite lattice differ from that on a large but finite lattice? A more fundamental question is

$$
Q_{1}: \quad h=h_{N}=h_{P}=h_{D} ?
$$

Here, $h=h(U)$ denotes the spatial entropy of $U$, the set (or a significant subclass) of stationary solutions (patterns) on an infinite lattice; and $h_{N}, h_{P}$, and $h_{D}$ represent the spatial entropy for the same class of solutions (patterns) which satisfy Neumann, periodic, and Dirichlet boundary conditions, respectively. These notations are precisely defined in section 2. $Q_{1}$ was posed by Afraimovich [2]. Let us provide a preliminary thought to this problem. In a lattice dynamical system, stationary solutions on a large lattice or infinite lattice can frequently be constructed in the following manner, cf. [29], [37]. First, one analyzes the existence of stationary solutions on a small lattice. The translation-invariant property (as explained in section 2) of the system is then used to attach the solutions that exist on small lattice compatibly and construct

\footnotetext{
* Received by the editors June 22, 1998; accepted for publication (in revised form) November 26, 1999; published electronically July 19, 2000. This research was supported in part by the National Science Council of Taiwan, Republic of China.

http://www.siam.org/journals/siap/61-1/34065.html

$\dagger^{\dagger}$ Department of Applied Mathematics, National Chiao-Tung University, 1001 Ta-Hsueh Road, Hsinchu, Taiwan, Republic of China (cwshih@math.nctu.edu.tw).
} 
stationary solutions on a large lattice or infinite lattice. Along this consideration, if the existence of the stationary state (solution) at a site of a lattice depends heavily on the states at its neighboring sites, then the total number of stationary solutions on the infinite lattice is expected to be lower. In this case, the entropy $h$ is likely to be zero. However, owing to this heavy dependence, boundary effect is also strong and the number of stationary solutions satisfying the boundary condition is restrained. And then $h=h_{B}=0, B=N, P, D$. On the other hand, consider a situation in which the existence of the state at a site depends weakly on the states at its neighboring sites. Under this circumstance, although more stationary solutions are likely available, the influence on the pattern forming from boundary conditions is also weaker. Therefore, an answer to $Q_{1}$ tending toward the positive is (more or less) conjectured, that is, $h=h_{B}$. However, a proof or an example with justification is necessary.

Throughout this paper, feasible solutions and patterns (corresponding to certain parameters) shall mean stable stationary solutions and patterns which exist for the system (with these parameters). For some fixed parameters, let $\Gamma_{\mathbf{k}}^{B}$ be the number of feasible patterns on a finite lattice of size $\mathbf{k}$, which satisfy the boundary condition $B$. Also, let $\Gamma^{\infty}$ be the number of feasible patterns on an infinite lattice, corresponding to the same parameters. Even if $Q_{1}$ is true, one does not expect, in general, that $\Gamma^{\infty}$ coincides with $\lim _{\mathbf{k} \rightarrow \infty} \Gamma_{\mathbf{k}}^{B}$. For example, suppose $\Gamma_{\mathbf{k}}$ is a constant multiple of $\Gamma_{\mathbf{k}}^{B}$ for each $\mathbf{k}$, where $\Gamma_{\mathbf{k}}$ is the number of patterns projected from the patterns on an infinite lattice onto a lattice of size $\mathbf{k}$. The spatial entropy defined through these two quantities are still identical, that is, $h=h_{B}$. However, the following question arises: How do the the solutions on finite lattices with a certain boundary condition approximate, in some norm, the solutions on an infinite lattice? Or, for each solution $u$ on an infinite lattice, can the projection of $u$ on the main central sites coincide with some solution on these sites, which satisfies certain boundary conditions? The former one is restated more precisely as follows.

$Q_{2}$ : Given a solution $u$ (or a pattern) on the infinite lattice $\mathbf{Z}^{d}$, does there exist a solution $u_{T}$ (or a pattern) on $T \subset \mathbf{Z}^{d}$, which satisfies certain a boundary condition, and $u_{T}$ approximates $u$ in some norm, as $T$ tends to $\mathbf{Z}^{d}$ ?

Basically, what is asked in $Q_{2}$ is rather strong. A positive answer to it requires that "every" feasible pattern on an infinite lattice can be approximated by feasible patterns on large finite lattices with boundary conditions. In addition, topology must be specified when considering whether or not answering one of the questions $Q_{1}$ and $Q_{2}$ implies an answer to another.

In the case of a one-dimensional (1- $d$ ) lattice, the generation of patterns for a lattice system can frequently be described by the so-called "transition matrix" $M$. Suppose the size of $T$ is $k$ (e.g., $T:=\{i \mid 1 \leq i \leq k\}$ ). The sum of all entries in $M^{k-1}$ gives the number of patterns on $T$; cf. [9], [35]. These patterns represent the patterns on $T$, projected from the feasible patterns on the infinite lattice, if the transition matrix is appropriately formulated. For such projected patterns, boundary conditions and the feasibility of patterns with respect to the imposed boundary conditions on $T$ are not considered. If a certain boundary condition is imposed, then the number of feasible patterns on $T$ may become the sum of only some of the entries in $M^{k-1}$ or even zero. The latter case, in which the projected patterns are usually very limited, is more likely to occur in the regime of pattern formation, whereas the former can occur in the regime of spatial chaos (as defined in section 2). In some cases, the number of feasible patterns equals a single entry of $M^{k-1}$.

The studies in influence of boundary conditions upon the solution structures of 
partial differential equations have been done by many scientists. For example, Mielke [34] has indicated that some typical patterns in Ginzburg-Landau equations are not dominated by boundary effects. Homotopy of boundary conditions has been used by Fiedler [23], Hale and Rocha [26], and Gardner [25] to study global attractors of a class of reaction-diffusion equations. These studies analyzed the detailed effect of boundary conditions on the structure of global attractors from different aspects. For example, Fiedler showed that the class of global attractors is independent of boundary conditions. If the equilibrium is nonhyperbolic and a bifurcation occurs, the bifurcation scenario such as the structure of attractors may vary with respect to boundary conditions. This has been observed by Dillon, Maini, and Othmer [21] in the study of pattern formation in generalized 1- $d$ Turing systems, and by Mei and Theil [33] in the analysis of steady state bifurcations, as well as by Holder and Schaeffer [28], and Schaeffer and Golubitsky [36] on mode-jumping of von Kármán equations.

Lattice dynamical systems can be found in many scientific models, including the models in chemical reactions [22], [31], material sciences [7], [18], biology [6], [30], image processing and pattern recognition [11], [14], [15], [16], [20], [24], [38].

This study shall attempt to derive the solutions for $Q_{1}$ and $Q_{2}$ in a cellular neural network (CNN) model. While aiming to achieve this goal, this work also obtains some interesting results for CNN itself, which can be regarded as an independent part of this paper. The CNN model we study is proposed by Chua and Yang in 1988; cf. [13]. Its applications in image processing and pattern recognition can be found in the above-mentioned references. This study focuses mainly on exploring how boundary conditions affect the pattern formation and spatial chaos for CNN. For this purpose, the pattern forming properties for CNN with a finite number of cells (CNN on a finite lattice) are also investigated. Therefore, our results also demonstrate how the boundary conditions affect the global attractor of CNN, which has practical implications for circuit design. The so-called mosaic patterns (solutions) in CNN are considered in our investigations. These mosaic solutions are all stable for CNN on a finite lattice and on an infinite lattice. Thus, the set of all mosaic solutions is contained in the global attractor of CNN. It will be seen how the attractor varies with respect to the parameters and different boundary conditions. Notably, the mosaic solutions are called stable system equilibrium points in Chua and Yang [13].

In a 1-d CNN, formation of mosaic patterns can be fully described via transition matrices. Also discussed herein is how the boundary conditions influence the transition matrices. $Q_{1}$ and $Q_{2}$ can then be resolved completely. Results in this study demonstrate that, although subtly, $Q_{1}$ is true, possibly owing to that the dimension is low and the impact from the boundary conditions is weak. In the two-dimensional $(2-d)$ case, we have counterexamples to $Q_{1}$, indicating that $h \neq h_{D}$ and $h \neq h_{N}$, respectively. As for the solution to $Q_{2}$, some cases (parameters) can always be found so that it is true or false, in $1-d$ and $2-d$.

Mosaic patterns have been studied in discrete reaction diffusion models [9], [10], [12], as well as in CNN [29], [37]. The considerations in these works centered around patterns on an infinite lattice. Results in this study are presented for the first time in the following aspects. A family of transition matrices is formulated. All mosaic patterns of $1-d$ CNN with a general $1 \times 3$ template, on an infinite lattice and on a finite lattice with boundary conditions can be generated from these matrices. Boundary conditions (on 1- $d$ and 2- $d$ ) have not been taken into account in the notion of spatial chaos, the formulation of a transition matrix and the existence of patterns on a finite lattice, in all the aforementioned studies [9], [10], [12], [29], [37]. 
The rest of this paper is organized as follows. Section 2 reviews the lattice dynamical system with some of its basic features. Two general propositions (2.1 and 2.2) are given which provide a criteria toward the positive answers to $Q_{1}$ and $Q_{2}$, respectively. We then focus our discussion on CNN. Section 3 describes some general properties of CNN. In section 4 , the solutions of $Q_{1}$ and $Q_{2}$ are derived for 1- $d$ CNN with general templates, using both the setting of transition matrix and Propositions 2.1 and 2.2. Finally, in section 5, the solutions of $Q_{1}$ and $Q_{2}$ are derived for 2- $d$ CNN with a two-parameter symmetric template, mainly using Propositions 2.1, 2.2. Taking this template and another horizontally symmetric template, some counterexamples are given.

2. General formulation and propositions. This study largely concerns itself with a class of lattice dynamical systems (LDS) which can be described as follows. Let $d \geq 1$ be an integer and $\Lambda$ be the $d$-dimensional integer lattice or a subset of it. That is, $\Lambda \subseteq \mathbf{Z}^{d} \subseteq \mathbf{R}^{d}$. The LDS considered herein is a system with continuous time, and the spatial variable takes values in the discrete lattice $\Lambda$. The state of the system is represented by the vector $\left\{u_{\alpha}\right\}_{\alpha \in \Lambda}$ with $u_{\alpha} \in \mathbf{R}$ for each $\alpha$. In the case $\Lambda=\mathbf{Z}^{d}$, the phase space $X$ is an infinite-dimensional Banach space. For example,

$$
X=\left\{u=\left\{u_{\alpha}\right\}: \alpha \in \mathbf{Z}^{d},\|u\|<\infty\right\}
$$

where $u_{\alpha} \in \mathbf{R}$ and the norm $\|\cdot\|$ could be $\|\cdot\|_{\ell^{\infty}}$, the $\ell^{\infty}$ norm or $\|\cdot\|_{\ell^{2}}$, the $\ell^{2}$ norm or $\|\cdot\|_{\ell_{q}^{2}}$, the $\ell_{q}^{2}$ norm. The $\ell_{q}^{2}$ norm is defined as follows:

$$
\|u\|_{\ell_{q}^{2}}=\left(\sum_{\alpha \in \mathbf{Z}^{d}} q^{-|\alpha|}\left|u_{\alpha}\right|^{2}\right)^{1 / 2},
$$

where $q>0$ is a fixed number, $|\alpha|=\max \left\{\left|\alpha_{l}\right|, l=1, \ldots, d\right\}$, and $\alpha=\left(\alpha_{1}, \ldots, \alpha_{d}\right) \in$ $\mathbf{Z}^{d}$. These spaces are represented by $X=\ell^{\infty}, \ell^{2}, \ell_{q}^{2}$, respectively. Notably, $\ell^{2}=\ell_{q}^{2}$ for $q=1$. A previous investigation has demonstrated that the norm $\|\cdot\|_{\ell_{q}^{2}}$ is quite useful in investigating a space-time chaos in LDS; cf. [3] and the references therein.

The LDS with continuous time can be expressed by

$$
d u / d t=\mathcal{F}(u(t)), \text { where } \mathcal{F}: X \rightarrow X
$$

Or in coordinate form

$$
\frac{d u_{\alpha}}{d t}=\mathcal{F}_{\alpha}\left(\left\{u_{\gamma}\right\}_{\gamma \in \Lambda}\right), \quad \alpha \in \Lambda
$$

Generally, if $\mathcal{F}$ is locally Lipschitz in $X$, the existence and uniqueness of the initial value problem for LDS hold; see [5], [8]. Furthermore, the systems of interest, for example, spatially discrete version of Allen-Cahn equation (cf. [7]) and CNNs, have the property of finite-range interaction. Let a finite subset $G \subseteq \mathbf{Z}^{d}$ be fixed (it describes the range of coupling). Then $\mathcal{F}$ can be written as

$$
\mathcal{F}(u)_{\alpha}=F\left(\left\{u_{\alpha+\tilde{\alpha}}\right\}_{\tilde{\alpha} \in G}\right), \quad \alpha \in \mathbf{Z}^{d},
$$

where $F:\left\{\left\{v_{\tilde{\alpha}}\right\}_{\tilde{\alpha} \in G} \mid v_{\tilde{\alpha}} \in \mathbf{R}\right\} \rightarrow \mathbf{R}$ is a smooth function. These systems also have the property of invariance under translations of the lattice. Let $\left\{S_{\gamma}\right\}_{\gamma \in \mathbf{z}^{d}}$ be the group 
of translations acting on the space $X$, which is defined by $\left(S_{\gamma} u\right)_{\alpha}=u_{\gamma+\alpha}$. A direct consequence of this invariance is

$$
u \in \mathcal{E}^{\infty} \Longleftrightarrow S_{\gamma} u \in \mathcal{E}^{\infty},
$$

where $\mathcal{E}^{\infty}$ is the set of all stationary solutions of (2.1). Further details can be found in [10]. Herein, we denote by $(\mathrm{LDS})_{\infty}$ the infinite-dimensional initial value problem for the LDS on $\mathbf{Z}^{d}$.

If $\Lambda$ is only a proper subset of $\mathbf{Z}^{d}$, then boundary conditions are natually imposed on the boundary of $\Lambda$ in $\mathbf{Z}^{d}$. For example, consider $\Lambda$ to be the following finite lattice $T_{\mathbf{k}}$ :

$$
T_{\mathbf{k}}=\left\{\left(\alpha_{1}, \alpha_{2}, \ldots, \alpha_{d}\right) \in \mathbf{Z}^{d}: 1 \leq \alpha_{l} \leq k_{l}\right\},
$$

where $\mathbf{k}=\left(k_{1}, \ldots, k_{d}\right)$ is a $d$-tuple of positive integers. The width of the frame of boundary sites is usually equal to the neighborhood size $r$. The latter one is related to the coupling range $G$. For instance, if $d=2, G=\{(i, j) \mid i, j=-1,0,1\}$, then $r=1$. In this case, the boundary sites are

$$
\mathbf{b}:=\left\{\left(k_{1}+1, j\right),(0, j),\left(i, k_{2}+1\right),(i, 0), 0 \leq i \leq k_{1}, 0 \leq j \leq k_{2}\right\} .
$$

The following three types of boundary conditions for LDS on $T_{\mathbf{k}}$ are considered:

- $(\mathrm{LDS})_{\mathbf{k}}-N$, LDS with Neumann boundary condition on $T_{\mathbf{k}}$;

- $(\mathrm{LDS})_{\mathbf{k}}-P$, LDS with periodic boundary condition on $T_{\mathbf{k}}$;

- $(\mathrm{LDS})_{\mathbf{k}}-D$, LDS with Dirichlet boundary condition on $T_{\mathbf{k}}$.

These boundary conditions are discrete analogues of the ones in PDEs; cf. [16]. For the case $d=2, r=1$, let us describe precisely these boundary conditions on $T_{\mathbf{k}}$. The Neumann boundary condition is the zero flux boundary condition. The states of the boundary sites are set equal to the states at the corresponding neighboring sites in $T_{\mathbf{k}}$. Namely, for $0 \leq i \leq k_{1}+1,0 \leq j \leq k_{2}+1$,

$$
\begin{array}{ll}
u_{k_{1}+1, j}=u_{k_{1}, j}, & u_{0, j}=u_{1, j}, \\
u_{i, k_{2}+1}=u_{i, k_{2}}, & u_{i, 0}=u_{i, 1} .
\end{array}
$$

The periodic boundary condition identifies the first and the last rows (respectively, columns) of the array $T_{\mathbf{k}}$, thereby forming a torus. Namely, for $0 \leq i \leq k_{1}+1,0 \leq$ $j \leq k_{2}+1$,

$$
\begin{array}{lll}
u_{1, j}=u_{k_{1}, j}, & u_{0, j}=u_{k_{1}-1, j}, & u_{2, j}=u_{k_{1}+1, j} \\
u_{i, 1}=u_{i, k_{2}}, & u_{i, 0}=u_{i, k_{2}-1}, & u_{i, 2}=u_{i, k_{2}+1} .
\end{array}
$$

The Dirichlet boundary condition means that certain boundary data (fixed constants) are prescribed on the boundary sites $\mathbf{b}$, that is, $u_{\mathbf{b}}=\hat{u}_{\mathbf{b}}:=\left\{\hat{u}_{i, j},(i, j) \in \mathbf{b}\right\}$.

Notably, LDS on $T_{\mathbf{k}}$ with any above boundary condition is a system of ODEs on a finite-dimensional phase space. Its existence and uniqueness theorems follow from the regular fundamental theorems of ODEs.

To illustrate the spatial complexity of $(\mathrm{LDS})_{\infty}$, the definition of spatial entropy is recalled. Let $\mathcal{A}$ be a finite set of elements (symbols) which are used to represent the patterns at each site on the lattice. Let $\mathcal{A}^{\mathbf{Z}^{d}}$ represent the set of all functions $y: \mathbf{Z}^{d} \rightarrow \mathcal{A}$. There is a natural projection

$$
\pi_{\mathbf{k}}: \mathcal{A}^{\mathbf{Z}^{d}} \rightarrow \mathcal{A}^{T_{\mathbf{k}}}
$$


given by restricting any $y \in \mathcal{A}^{\mathbf{Z}^{d}}$ to finite subset $T_{\mathbf{k}}$. Assume that $\mathcal{U}$ is a translational invariant subset of stationary solutions $\mathcal{E}^{\infty}$ in $(\mathrm{LDS})_{\infty}$, or of $\mathcal{A}^{\mathbf{Z}^{d}}$, which represents a class of patterns in $(\mathrm{LDS})_{\infty}$. Set

$$
\Gamma_{\mathbf{k}}(\mathcal{U}):=\operatorname{card}\left(\pi_{\mathbf{k}}(\mathcal{U})\right) .
$$

This quantity refers to the number of distinct patterns among the elements of $\mathcal{U}$, when restricting one's observation to the subset $T_{\mathbf{k}}$. The spatial entropy $h(\mathcal{U})$ is defined as

$$
h(\mathcal{U}):=\lim _{\mathbf{k} \rightarrow \infty} \frac{1}{k_{1} k_{2} \cdots k_{d}} \ln \Gamma_{\mathbf{k}}(\mathcal{U}) .
$$

On the other hand, when considering the problems $(\mathrm{LDS})_{\mathbf{k}}-B$ on finite lattice $T_{\mathbf{k}}$, boundary condition $B$ should be taken into account. Denote by $\mathcal{U}_{\mathbf{k}}^{B}(B=N, P, D)$ a class of patterns (or stationary solutions) for (LDS) $)_{\mathbf{k}}-B$. We can define the spatial entropy, depending on boundary condition $B$, by using $\Gamma\left(\mathcal{U}_{\mathrm{k}}^{B}\right):=\operatorname{card}\left(\mathcal{U}_{\mathrm{k}}^{B}\right)$ in (2.3). The spatial entropy defined in this setting is denoted by $h_{N}=h_{N}\left(\mathcal{U}^{N}\right), h_{P}=$ $h_{P}\left(\mathcal{U}^{P}\right), h_{D}=h_{D}\left(\mathcal{U}^{D}\right)$, corresponding to boundary condition $N$ (Neumann), $P$ (periodic), and $D$ (Dirichlet), respectively.

To discuss approximating a solution on infinite lattice by a solution on a finite lattice, there can be various considerations. One consideration is to identify the coincidence on the main central sites for a solution on an infinite lattice and a corresponding solution on a (large) finite lattice with boundary conditions. This is the main content in the following conditions: $\left(H_{1}\right)$ and $\left(H_{2}\right)$. Alternatively, a suitable norm can be used to measure the difference between two corresponding solutions. Which norm is appropriate depends on the nature of the problem (LDS). To be more explicit in this consideration, assume that $u=\left\{u_{i, j}\right\}$ is a solution on infinite lattice $\mathbf{Z}^{2}$ and $u_{T}$ is a solution on finite lattice $T=T_{\mathbf{k}} \subset \mathbf{Z}^{2}$. The expression $\left\|u-u_{T}\right\|$ is meaningful only if $u, u_{T}$ belong to the same space. However, $u \in X=\ell^{\infty}=\ell^{\infty}\left(\mathbf{Z}^{2}\right)$ or $\ell_{q}^{2}=\ell_{q}^{2}\left(\mathbf{Z}^{2}\right)$, whereas $u_{T} \in\left\{v=\left\{v_{i, j}\right\}_{(i, j) \in T}: v_{i, j} \in \mathbf{R}\right\}$. Therefore, one has to be more specific in discussing the approximation in terms of the norm. An option is to consider $\left\|\pi_{\mathbf{k}}(u)-u_{T}\right\|_{\ell^{\infty}(T)}\left(\right.$ or $\left.\left\|\pi_{\mathbf{k}}(u)-u_{T}\right\|_{\ell_{q}^{2}(T)}\right)$, where $\|v\|_{\ell^{\infty}(T)}=\sup \left\{\left|v_{i, j}\right|:(i, j) \in T\right\}$ and $\|v\|_{\ell_{q}^{2}(T)}^{2}=\sum_{(i, j) \in T} q^{-|(i, j)|}\left|v_{i, j}\right|^{2}$. Equivalently, $u_{T}$ can be extended to an element $\tilde{u}_{T}$ that is defined on $\mathbf{Z}^{2}$ by setting $\left(\tilde{u}_{T}\right)_{i, j}=u_{i, j}$ for $(i, j) \in \mathbf{Z}^{2} \backslash T$ and, then, considering $\left\|u-\tilde{u}_{T}\right\|_{\ell^{\infty}}\left(\right.$ or $\left.\left\|u-\tilde{u}_{T}\right\|_{\ell_{q}^{2}}\right)$. For $\|\cdot\|=\|\cdot\|_{\ell^{\infty}}$ or $\|\cdot\|_{\ell_{q}^{2}}$, we state $Q_{2}$ in this consideration precisely as follows:

Let $u \in \mathcal{U} \subseteq \mathcal{E}^{\infty}$. Given $\varepsilon>0$, do there exist a positive integer $\tilde{k}$ and $\tilde{v^{\mathbf{k}}} \in X$ for every $d$-tuple of positive integers $\mathbf{k}=\left(k_{1}, \ldots, k_{d}\right)$ with every $k_{l}>\tilde{k}$ such that $\pi_{\mathbf{k}}(\tilde{v} \mathbf{k})$

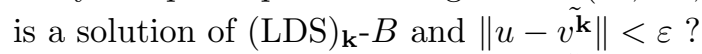

In this setting, obtaining this $v^{\mathbf{k}} \in X$ amounts to obtaining a solution $v^{\mathbf{k}}$ of (LDS) $)_{\mathbf{k}}-B$ such that $\left\|\pi_{\mathbf{k}}(u)-v^{\mathbf{k}}\right\|_{\ell^{\infty}(T)}$ or $\left\|\pi_{\mathbf{k}}(u)-v^{\mathbf{k}}\right\|_{\ell_{q}^{2}(T)}<\varepsilon$. If $\tilde{k}$ is independent of $u$, the approximation in $Q_{2}$ is said to be uniform. Notably, $Q_{2}$ is discussed in the context of stationary solutions (not patterns in symbols) of (LDS) $)_{\mathbf{k}}-B$ and (LDS) $)_{\infty}$. In the following, we give two criteria which indicate positive answers to $Q_{1}$ and $Q_{2}$, respectively. Assume that the patterns on the boundary sites (as $\mathbf{b}$ in (2.2) for case $d=2$ ), which are reflected from previously described boundary conditions, are the same elements (symbols) that represent the patterns in $\mathcal{U}$ at each site. The following notations will be used. By $\mathbf{k}>s$, we mean $k_{l}>s$, for all $l=1, \ldots, d$ and by $\pi_{\mathbf{k}-s}$ we mean the projection onto $T_{\mathbf{k}-s}$, where $T_{\mathbf{k}-s}=\left\{\left(\alpha_{1}, \alpha_{2}, \ldots, \alpha_{d}\right) \in \mathbf{Z}^{d}: 1+s \leq \alpha_{l} \leq\right.$ $\left.k_{l}-s\right\} \subset T_{\mathbf{k}}$. The basic conditions are as follows: 
$\left(H_{1}\right) \quad$ There is a fixed positive integer $s$ such that for every $u \in \mathcal{U}$ and for each $\mathbf{k}>s$, there exists $w^{\mathbf{k}} \in X$ (or $\mathcal{A}^{\mathbf{Z}^{d}}$ ) with $\pi_{\mathbf{k}-s}\left(w^{\mathbf{k}}\right)=\pi_{\mathbf{k}-s}(u)$ and $\pi_{\mathbf{k}}\left(w^{\mathbf{k}}\right)$ is a solution (or pattern) of (LDS) $)_{\mathbf{k}}-B$.

$\left(H_{2}\right) \quad$ For each $u \in \mathcal{U} \subseteq \mathcal{E}^{\infty}$, there are constants $c_{1}>0, c_{2}>1$ such that for each $d$-tuple of integers $\mathbf{k}$, there exists a solution $v^{\mathbf{k}}$ of $(\operatorname{LDS})_{\mathbf{k}}-B$ with $\left|\left(v^{\mathbf{k}}\right)_{\alpha}\right|<c_{1}$, $\alpha \in T_{\mathbf{k}}$ and $\pi_{\mathbf{k}-s}\left(v^{\mathbf{k}}\right)=\pi_{\mathbf{k}-s}(u)$ for some integer $s<\left(1 / c_{2}\right) \cdot \min \left\{k_{l}: l=1, \ldots, d\right\}$.

Notably, $\left(H_{2}\right)$ implies that $\|u\|_{\ell \infty}<c_{1}$. In $\left(H_{1}\right), s$ does not depend on $u$, while $s$ and $c_{1}, c_{2}$ are allowed to depend on $u$ in $\left(H_{2}\right)$. Formulation of the conditions in $\left(H_{1}\right),\left(H_{2}\right)$ involves the notion of boundary reconstruction scheme, as described later for CNN in sections 4 and 5. Basically, for $u \in \mathcal{U}$, if we can reconstruct the solutions (or output patterns) on the sites in and near the boundary of $T_{\mathbf{k}}$ for $\pi_{\mathbf{k}}(u)$ to fit the boundary condition, then we have positive answers to $Q_{1}$ and $Q_{2}$. If this can be achieved for certain parameters, then one establishes a sense that the strength of effect from boundary conditions is weak for these parameters. Assume that the range of interaction (e.g., the radius of aforementioned $G$ ) has length $r$. In the propositions, recall that $\Gamma_{\mathbf{k}}(\mathcal{U})=\operatorname{card}\left(\pi_{\mathbf{k}}(\mathcal{U})\right)=: \Gamma_{\mathbf{k}}^{\infty}$ denotes the number of solutions (patterns) on $T_{\mathbf{k}}$ projected from $\mathcal{U}$ and $\Gamma\left(\mathcal{U}_{\mathbf{k}}^{B}\right)=\operatorname{card}\left(\mathcal{U}_{\mathbf{k}}^{B}\right)=: \Gamma_{\mathbf{k}}^{B}$ represents the number of solutions (patterns) in $\mathcal{U}_{\mathbf{k}}^{B}$.

Proposition 2.1. Let $\mathcal{U}$ be a translational invariant subset of stationary solutions $\mathcal{E}^{\infty}$, or of $\mathcal{A}^{\mathbf{Z}^{d}}$. Assume $\left(H_{1}\right)$ and $\Gamma_{\mathbf{k}}^{B} \leq p^{c} \cdot \Gamma_{\mathbf{k}-r}^{\infty}$ for some $p>0$ and $c=c(\mathbf{k})$ with $\lim _{\mathbf{k} \rightarrow \infty}\left[c /\left(k_{1} k_{2} \cdots k_{d}\right)\right]=0$, then $h=h_{B}$, where $B=N$ or $P$ or $D$.

Proposition 2.2. Consider $X=\ell_{q}^{2}$ with $q>1$. Let $\mathcal{U}$ be a translational invariant subset of $\mathcal{E}^{\infty}$, then $\left(H_{2}\right)$ implies that $Q_{2}$ is true.

Proposition 2.2 is formulated for the phase space $X=\ell_{q}^{2}$ with $q>1$, in general cases. In fact, $Q_{2}$ is true for $\mathrm{CNN}$ on $X=\ell^{\infty}$ with $B=N, P, D$ for certain parameter regions. This space is more practical for $\mathrm{CNN}$ from the application viewpoint. In sections 4 and 5, we elaborate on these results. The proofs of Propositions 2.1 and 2.2 are given successively as follows.

Proof of Proposition 2.1. We show only the case $d=2$. Note that $\Gamma_{\mathbf{k}}^{B} \geq \Gamma_{\mathbf{k}-s}^{\infty}$, according to $\left(H_{1}\right)$. Thus, on the one hand,

$$
\begin{aligned}
h_{B}\left(\mathcal{U}^{B}\right) & =\lim _{\mathbf{k} \rightarrow \infty} \frac{1}{k_{1} k_{2}} \ln \Gamma_{\mathbf{k}}^{B} \\
& \geq \lim _{\mathbf{k} \rightarrow \infty} \frac{1}{k_{1} k_{2}} \ln \Gamma_{\mathbf{k}-s}^{\infty} \\
& =\lim _{\mathbf{k} \rightarrow \infty} \frac{\left(k_{1}-2 s\right)\left(k_{2}-2 s\right)}{k_{1} k_{2}} \frac{\ln \Gamma_{\mathbf{k}-s}^{\infty}}{\left(k_{1}-2 s\right)\left(k_{2}-2 s\right)} \\
& =h(\mathcal{U}),
\end{aligned}
$$

and, on the other hand,

$$
\begin{aligned}
h_{B}\left(\mathcal{U}^{B}\right) & =\lim _{\mathbf{k} \rightarrow \infty} \frac{1}{k_{1} k_{2}} \ln \Gamma_{\mathbf{k}}^{B} \\
& \leq \lim _{\mathbf{k} \rightarrow \infty} \frac{1}{k_{1} k_{2}} \ln \left(p^{c} \cdot \Gamma_{\mathbf{k}-r}^{\infty}\right) \\
& =\lim _{\mathbf{k} \rightarrow \infty} \frac{\left(k_{1}-2 r\right)\left(k_{2}-2 r\right)}{k_{1} k_{2}} \frac{c \ln p+\ln \Gamma_{\mathbf{k}-r}^{\infty}}{\left(k_{1}-2 r\right)\left(k_{2}-2 r\right)} \\
& =h(\mathcal{U}) .
\end{aligned}
$$


Proof of Proposition 2.2. Assume that $u \in \mathcal{U}$. Given $\varepsilon>0$, let $m$ be a positive integer such that

$$
\max \left\{\left(\sum_{|\alpha|>m} q^{-|\alpha|}\left|u_{\alpha}\right|^{2}\right)^{1 / 2},\left(\sum_{|\alpha|>m} q^{-|\alpha|} c_{1}^{2}\right)^{1 / 2}\right\}<\varepsilon / 2 .
$$

Choose $\tilde{k}>m c_{2} /\left(c_{2}-1\right)$. For any $d$-tuple of positive integers $\mathbf{k}$ with $k_{l}>\tilde{k}, l=$ $1, \ldots, d$, let $v^{\mathbf{k}}, s=s(\mathbf{k})$ be as in the assumption $\left(H_{2}\right)$. Note that $k_{l}-s>k_{l}\left(c_{2}-\right.$ $1) / c_{2}>m$. Set $\left(v^{\tilde{\mathbf{k}}}\right)_{\alpha}=u_{\alpha}$ for $\alpha \in\left(\mathbf{Z}^{d} \backslash T_{\mathbf{k}}\right),\left(\tilde{v^{\mathbf{k}}}\right)_{\alpha}=\left(v^{\mathbf{k}}\right)_{\alpha}$ for $\alpha \in T_{\mathbf{k}}$. Then $\tilde{v}^{\mathbf{k}} \in X$, $\pi_{\mathbf{k}}(\tilde{v} \mathbf{k})$ is a solution of (LDS) $)_{\mathbf{k}}-B$, and $\left\|u-v^{\tilde{k}}\right\|_{\ell_{q}^{2}}<\varepsilon$. The proof is completed.

In sections 4 and 5 , we locate the parameters for CNN for which the boundary reconstruction schemes can be developed and, in doing so, the conditions $\left(H_{1}\right),\left(H_{2}\right)$ can be fulfilled. Thereafter, we can answer $Q_{1}, Q_{2}$ for CNN via Propositions 2.1, 2.2.

In the proof of Proposition 2.1, the equality $h_{B}\left(\mathcal{U}^{B}\right)=h(\mathcal{U})$ comes from two inequalities. One follows from $\left(H_{1}\right)$ and the other follows from the assumption $\Gamma_{\mathbf{k}}^{B} \leq$ $p^{c} \cdot \Gamma_{\mathbf{k}-r}^{\infty}$. Let us give the motivation for this assumption. In counting the number of feasible patterns on $T_{\mathbf{k}}$, there are three quantities, namely $\Gamma_{\mathbf{k}}^{\infty}, \Gamma_{\mathbf{k}}^{B}$, and $\Gamma_{\mathbf{k}}$. The first two were introduced earlier. The last one is the number of patterns on $T_{\mathbf{k}}$, without considering any boundary condition. Although the feasibility for such kinds of patterns is not well defined, they can still be obtained from a construction process: attaching feasible patterns on a lattice of smaller size compatibly to form patterns on a larger lattice, as is the methodology in [29], [37]. However, one knows for sure only that $\Gamma_{\mathbf{k}}^{\infty} \leq \Gamma_{\mathbf{k}}, \Gamma_{\mathbf{k}}^{B} \leq \Gamma_{\mathbf{k}}$, and not the relation between $\Gamma_{\mathbf{k}}^{\infty}$ and $\Gamma_{\mathbf{k}}^{B}$. Under normal circumstances, the assumption $\Gamma_{\mathbf{k}}^{B} \leq p^{c} \cdot \Gamma_{\mathbf{k}-r}^{\infty}$ in Proposition 2.1 or even $\Gamma_{\mathbf{k}}^{B} \leq \Gamma_{\mathbf{k}}^{\infty}$ is expected to hold, at least for some kind of boundary condition $B$. For example, in the case $d=2$, fix $\mathbf{k}$, every site of $T_{\mathbf{k}-r}$ is interior in $T_{\mathbf{k}}$. The number of patterns on $T_{\mathbf{k}-r}$, without considering any boundary condition, is expected to be the same as $\Gamma_{\mathbf{k}-r}^{\infty}$. The number of sites on $T_{\mathbf{k}}$ and outside $T_{\mathbf{k}-r}$ is $2 \cdot r \cdot k_{1}+2 \cdot r \cdot k_{2}-4 \cdot r^{2}=: c_{r}$. In addition, the maximal possible number of patterns on these sites is $p^{c_{r}}$, where $p=\operatorname{card}(\mathcal{A})$. Thus, $\Gamma_{\mathbf{k}}^{B} \leq p^{c_{r}} \cdot \Gamma_{\mathbf{k}-r}^{\infty}$. Moreover, some boundary condition should exist so that pattern forming is no less restrictive in the case with that boundary condition imposed than the case which requires extending the patterns to infinite lattice. In the following discussion of mosaic patterns in CNN, $\Gamma_{\mathbf{k}}^{B} \leq p^{c} \cdot \Gamma_{\mathbf{k}-r}^{\infty}$ is satisfied for the boundary conditions $B=N, P$, and $D$.

Concerning the implication from answering one of the questions $Q_{1}$ and $Q_{2}$ to answering another, we make the following remarks. Consider $X=\ell_{q}^{2}, q>1$, and $\mathcal{U} \subseteq \mathcal{E}^{\infty}$. On the one hand, $\left(H_{1}\right)$ implies that $Q_{2}$ is true in the uniform sense if we further assume that in $\left(H_{1}\right)$, for each $u$, the corresponding $w^{\mathbf{k}}$ is uniformy bounded for all $\mathbf{k}$. On the other hand, a positive answer to $Q_{2}$ does not imply positive answer to $Q_{1}$. This can be seen by respecting $\left(H_{1}\right),\left(H_{2}\right)$ and the proofs of Propositions 2.1 and 2.2. Indeed, in $\left(H_{1}\right), s$ has to be a fixed integer for all $u \in \mathcal{U}$, which is needed in the proof of Proposition 2.1, whereas $s$ can depend on each $u \in \mathcal{U}$ in the proof of Proposition 2.2.

3. Cellular neural networks. A CNN is a large array of nonlinear analogue circuit, which is made of only locally connected cells. We consider the CNN model proposed by Chua and Yang in 1988; cf. [13], [14]. In this section, we present the equations of the model on the integer lattice in two dimensions. Some fundamental dynamic properties for this CNN model are summarized and verified. To our 
knowledge, previous literature has not derived these properties. The definitions and notations associated with the stationary solutions of CNN are also provided. We then discuss the model on finite lattice along with consideration of the boundary conditions mentioned in section 2. The stationary equation for CNN on 1- $d$ lattice is presented in the next section.

Consider CNN on $\mathbf{Z}^{2}$, under space-invariant coupling and without an input control term. The circuit equation of a cell is

$$
\frac{d x_{i, j}}{d t}=-x_{i, j}+z+\sum_{|k| \leq 1,|\ell| \leq 1} a_{k, \ell} f\left(x_{i+k, j+\ell}\right), \quad(i, j) \in \mathbf{Z}^{2}
$$

Herein, the node voltage $x_{i, j}$ at $(i, j)$ is called the state of the cell at $(i, j) . z$ is an independent current source, which is called a bias term. The output function $f$ (a nonlinearity) is given by

$$
f(\xi)=\frac{1}{2}(|\xi+1|-|\xi-1|)
$$

The model (3.1) we consider has coupling range $N_{1}(i, j)$ for the cell at $(i, j)$. Here,

$$
N_{1}(i, j)=\left\{(k, \ell) \in \mathbf{Z}^{2} \mid \max \{|k-i|,|\ell-j|\} \leq 1\right\} .
$$

The elements in $N_{1}(i, j)$ are called the nearest and the next-nearest neighbors of $(i, j)$. The real numbers $a_{k, \ell},|k|,|\ell| \leq 1$ describe the coupling weights between cells. They can be arranged into a $3 \times 3$ matrix

$$
A:=\left[\begin{array}{ccc}
a_{-1,1} & a_{0,1} & a_{1,1} \\
a_{-1,0} & a_{0,0} & a_{1,0} \\
a_{-1,-1} & a_{0,-1} & a_{1,-1}
\end{array}\right] .
$$

Such a matrix is called a (space-invariant) template. Furthermore, $A$ is called symmetric if $a_{k, \ell}=a_{-k,-\ell}$. This symmetry notion represents symmetric coupling weights between cells.

The dynamical system (3.1) on an infinite lattice is an infinite system of ODEs. Equation (3.1) can be written in the form

$$
\frac{d x}{d t}=\mathcal{F}(x), \quad x \in X,
$$

where

$$
\begin{aligned}
\mathcal{F}_{i, j}(x) & =F\left(\left\{x_{i+k, j+\ell}\right\}_{(k, \ell) \in N_{1}(0,0)}\right) \\
F\left(\left\{x_{k, \ell}\right\}_{(k, \ell) \in N_{1}(0,0)}\right) & :=-x_{0,0}+z+\sum_{|k| \leq 1,|\ell| \leq 1} a_{k, \ell} f\left(x_{k, \ell}\right) .
\end{aligned}
$$

Proposition 3.1. Let $X=\ell^{\infty}$ or $\ell_{q}^{2}, q>1$.

(i) The vector field $\mathcal{F}: X \rightarrow X$ is Lipschitz.

(ii) On $X$, the initial value problem for (3.1) is well posed, that is, for each $x_{0} \in X$, there exists a unique solution $x(t)$ with $x(0)=x_{0}$ for forward and backward time $t$ near $t=0$.

(iii) (3.1) is dissipative and every solution of it exists globally in time. 
Proof. We only present the $2-d$ case.

(i) Let $\hat{a}=\max \left\{\left|a_{k, \ell}\right|: k, \ell=-1,0,1\right\}$. Then $\left|\mathcal{F}_{i, j}(x)\right| \leq\left|x_{i, j}\right|+|z|+9 \hat{a}$. This verifies that $\mathcal{F}$ maps $X$ into $X$ for $X=\ell^{\infty}$. It can also be verified that $\mathcal{F}$ maps $\ell_{q}^{2}$ into $\ell_{q}^{2}$ for $q>1$. This assertion does not hold for $0<q \leq 1\left(\ell_{q}^{2}=\ell^{2}\right.$ as $\left.q=1\right)$. Recall the definition of $|(i, j)|=\max \{|i|,|j|\}$ in section 2 . To verify the Lipschitz condition for $\mathcal{F}$, the following computations are elaborated on. First, $f$ is Lipschitz. Indeed, $|f(\xi)-f(\eta)| \leq|\xi-\eta|$. Using this fact, we have

$$
\begin{aligned}
\left|\mathcal{F}_{i, j}(x)-\mathcal{F}_{i, j}(w)\right| & =\left|w_{i, j}-x_{i, j}+\sum_{|k| \leq 1,|\ell| \leq 1} a_{k, \ell}\left[f\left(x_{i+k, j+\ell}\right)-f\left(w_{i+k, j+\ell}\right)\right]\right| \\
& \leq\left|w_{i, j}-x_{i, j}\right|+\hat{a} \sum_{|k| \leq 1,|\ell| \leq 1}\left|x_{i+k, j+\ell}-w_{i+k, j+\ell}\right| \\
& \leq(9 \hat{a}+1)\|x-w\|_{\ell \infty} .
\end{aligned}
$$

Since this is true for every $(i, j)$, it follows that

$$
\|\mathcal{F}(x)-\mathcal{F}(w)\|_{\ell^{\infty}} \leq(9 \hat{a}+1)\|x-w\|_{\ell^{\infty}} .
$$

For $X=\ell_{q}^{2},(3.2)$ yields that

$$
\begin{aligned}
\left|\mathcal{F}_{i, j}(x)-\mathcal{F}_{i, j}(w)\right|^{2} & \leq\left\{\left|w_{i, j}-x_{i, j}\right|+\hat{a} \sum_{|k| \leq 1,|\ell| \leq 1}\left|x_{i+k, j+\ell}-w_{i+k, j+\ell}\right|\right\}^{2} \\
& \leq 10\left\{\left|w_{i, j}-x_{i, j}\right|^{2}+\hat{a}^{2} \sum_{|k| \leq 1,|\ell| \leq 1}\left|x_{i+k, j+\ell}-w_{i+k, j+\ell}\right|^{2}\right\} .
\end{aligned}
$$

Note that $q^{-|(i, j)|}=q \cdot q^{-|(i+k, j+\ell)|}$ or $(1 / q) \cdot q^{-|(i+k, j+\ell)|}$ for $k, \ell=-1,1$. Therefore, for $q>1$,

$$
\begin{aligned}
\|\mathcal{F}(x)-\mathcal{F}(w)\|_{\ell_{q}^{2}}^{2} & =\sum_{(i, j) \in \mathbf{Z}^{2}} q^{-|(i, j)|}\left|\mathcal{F}_{i, j}(x)-\mathcal{F}_{i, j}(w)\right|^{2} \\
& \leq \sum_{(i, j) \in \mathbf{Z}^{2}} 10 q^{-|(i, j)|}\left\{\left|w_{i, j}-x_{i, j}\right|^{2}+\hat{a}^{2} \sum_{|k| \leq 1,|\ell| \leq 1}\left|x_{i+k, j+\ell}-w_{i+k, j+\ell}\right|^{2}\right\} \\
& \leq 10\|x-w\|_{\ell_{q}^{2}}^{2}+10 \hat{a}^{2} \sum_{(i, j) \in \mathbf{Z}^{2}} \sum_{|k| \leq 1,|\ell| \leq 1} q \cdot q^{-|(i+k, j+\ell)|}\left|x_{i+k, j+\ell}-w_{i+k, j+\ell}\right|^{2} \\
& =10\|x-w\|_{\ell_{q}^{2}}^{2}+10 \hat{a}^{2} q \sum_{|k| \leq 1,|\ell| \leq 1} \sum_{(i, j) \in \mathbf{Z}^{2}} q^{-|(i+k, j+\ell)|}\left|x_{i+k, j+\ell}-w_{i+k, j+\ell}\right|^{2} \\
& =10\left(9 \hat{a}^{2} q+1\right)\|x-w\|_{\ell_{q}^{2}}^{2} .
\end{aligned}
$$

This verifies that $\mathcal{F}$ is Lipschitz on $X=\ell_{q}^{2}, q>1$.

(ii) The existence and uniqueness proof for finite-dimensional ODEs can be carried over to our infinite-dimensional systems here. Discussions for these fundamental properties in a general setting can be found in [5], [9].

(iii) It can be concluded by similar arguments as in [5] that the evolution operator for (3.1) exists for all $t \in \mathbf{R}$. In fact, for each $(i, j)$

$$
\mathcal{F}_{i, j}(x) x_{i, j}<0 \text { if }\left|x_{i, j}\right|>9 \hat{a}+|z| .
$$


Let $x_{0}$ be an arbitrary point in $X$, then the solution $\phi\left(t, x_{0}\right)$ of $(3.1)$ satisfies

$$
\left\|\phi\left(t, x_{0}\right)\right\|_{\ell^{\infty}} \leq \max \left\{2(9 \hat{a}+|z|),\left\|x_{0}\right\|_{\ell^{\infty}}\right\}
$$

Thus, every local (in time) solution can be extended. Equation (3.3) also implies that the bounded set $\left\{x \in X:\|x\|_{\ell^{\infty}} \leq 2(9 \hat{a}+|z|)\right\}$ attracts every point of $X$, therefore (3.1) is dissipative. A similar conclusion holds for $X=\ell_{q}^{2}, q>1$. Details regarding the notion of dissipative dynamical system can be found in [27]. This completes the proof.

Let $x=\left\{x_{i, j}\right\}$ be a stationary solution of (3.1). The associated output $y=$ $\left\{y_{i, j}\right\}=\left\{f\left(x_{i, j}\right)\right\}$ is called a (stationary) pattern. The stationary solutions and patterns can be classified into four types: mosaic, defect, interior, and transitional, as defined in [29], [37]. Herein, the mosaic and the defect ones on the 2-d lattice are recalled. They can be easily generalized to another dimension.

Definition 3.2. A stationary solution $x=\left\{x_{i, j}\right\}$ of (3.1) is called nontransitional if $\left|x_{i, j}\right| \neq 1$ for all $(i, j) \in \mathbf{Z}^{2}$. In particular, $x$ is called a mosaic solution if $\left|x_{i, j}\right|>1$ for all $(i, j) \in \mathbf{Z}^{2}$. Its associated pattern is called a mosaic pattern. If $\left|x_{i, j}\right| \neq 1$ for all $(i, j) \in \mathbf{Z}^{2}$ and there are $(m, n)$ and $(k, \ell)$ such that $\left|x_{m, n}\right|<1$ and $\left|x_{k, \ell}\right|>1$, then $x$ and $y=\left\{f\left(x_{i, j}\right)\right\}$ are called, respectively, a defect solution and a defect pattern.

This study focuses mainly on mosaic solutions and patterns. For generic parameters, an above-mentioned pattern, if it exists, corresponds to an isolated equilibrium of (3.1). Notably, our mosaic pattern takes value (pixel value in image processing) 1 or -1 at each $(i, j)$, whereas the mosaic pattern in Chow, Mallet-Paret, and Van Vleck [9] takes value 1 or 0 or -1 . Notice that the global mosaic solutions, an essential type of equilibrium in CNN, do not belong to $\ell_{q}^{2}, 0<q \leq 1$. For this and for the reason in Proposition 3.1, it is inappropriate to formulate CNN on $X=\ell_{q}^{2}, 0<q \leq 1\left(\ell_{q}^{2}=\ell^{2}\right.$, if $q=1$ ).

DEFINITION 3.3. $x$ is called a global solution if it is a stationary solution of (3.1) on $\mathbf{Z}^{2}$. In this case, $y\left(=\left\{f\left(x_{i, j}\right)\right\}\right)$ is called a global pattern. Given any (proper) subset $T \subseteq \mathbf{Z}^{2}, x\left(\equiv x_{T}\right)$ is called a local solution if $x_{T}$ is a restriction of some global solution on $T$. Similarly, $y\left(\equiv y_{T}\right)$ is called a local pattern if it is an output of some (local) solution $x_{T}$ on $T . A$ set $T \subset \mathbf{Z}^{2}$ is called basic with respect to the template $A$ if $T=\left\{(i+k, j+\ell) \in N_{1}(i, j) \mid a_{k, \ell} \neq 0\right\}$ for some $(i, j) \in \mathbf{Z}^{2}$. A basic (mosaic) pattern $y$ is a local mosaic pattern defined on the basic set.

Our recipe for finding the global patterns or local patterns is to attach the patterns on a smaller lattice compatibly and construct patterns on a larger lattice, as found in [29], [37]. The global and local mosaic patterns are constructed from the basic patterns. Following from our partitioning of parameters (note: the next two sections provide more details), the set of "tentative" basic patterns can be characterized with respect to parameter subregions. The set of "tentative" basic patterns contains two types of patterns defined on basic sets. Some of these patterns can appear only on the cells near the boundary of a finite lattice. These are referred to herein as boundary basic patterns. If a tentative basic pattern can be expanded by attaching to it some tentative basic patterns (including itself) in all directions (east, west, south, north in the 2- $d$ case) to form a global pattern, then this pattern is called a basic (mosaic) pattern, in respecting our Definition 3.3.

The term "feasible" generally refers to specifying "stable" stationary solutions; cf. [29]. According to a previous investigation, -1 is the only eigenvalue for the linearization of $\mathcal{F}$ at a mosaic solution. Therefore every global mosaic solution (pattern) is stable in the spaces $X=\ell^{\infty}, \ell_{q}^{2}, q>1$. In this study, since the solutions and patterns 
we are discussing are already stable, "feasible" solutions and patterns (corresponding to certain parameters) are adopted to emphasize the existence of the stable stationary solutions and patterns for the system (with these parameters).

The following dynamic properties for $\mathrm{CNN}$ on a finite lattice can be found in [13], [32]. If the parameters are given, every solution $x(t)$ of CNN is bounded for all time $t \geq 0$. Furthermore, if the template is symmetric, every orbit converges to an equilibrium. In addition, with further restriction on the central element of template $\left(a_{0,0}>1\right.$ in (3.1)), almost every orbit tends to a mosaic solution.

Definition 3.4. For any two integers $k<l$, denote $I[k, l]=\{k, k+1, \ldots, l\}$, the set of all integers that are no smaller than $k$ and no greater than $l$.

DEFINITION 3.5. Let $\mathcal{U}$ be a translation-invariant set of feasible global mosaic patterns. $\mathcal{U}$ is said to exhibit spatial chaos if the spatial entropy $h(\mathcal{U})$ is greater than zero. Otherwise, we say $\mathcal{U}$ exhibits pattern formation.

Next, we discuss CNN on a finite lattice $T$. Every cell of the CNN has the same neighborhood of interaction except the ones located on the edge of $T$. It was suggested in [17] that one can surround the rectangular array with boundary cells to compensate for the absent neighbors of these cells. The output for each of these boundary cells is chosen to be between -1 and 1 (in most applications, it has been set to zero) and remains constant with respect to time $t$. These are the boundary conditions of Dirichlet type. There is also a design of circular array; see [39]. In that proposed boundary condition, two edges of a 1- $d$ array are connected to form a circular circuit. Our periodic boundary condition fits into this setting. In the remaining discussion of patterns on a finite lattice, we shall consider patterns on a square lattice $T=T_{k}:=\left\{(i, j) \in \mathbf{Z}^{2} \mid-k \leq i, j \leq k\right\}$ in the 2-d case and $T=T_{k}:=\left\{i \in \mathbf{Z}^{\mathbf{1}} \mid-k \leq i \leq k\right\}$ in the $1-d$ case. This differs from $T_{\mathbf{k}}$ in section 2, and is merely for convenience of discussion. The Dirichlet boundary value problems of CNN on $T_{k}$ can be further classified into two types. Let $(\mathrm{CNN})_{k}-D_{1}$ denote the one with saturated boundary data. That is, each boundary cell has an output of magnitude equal to one. Let $(\mathrm{CNN})_{k}-D_{0}$ denote the one with defect boundary data. That is, each boundary cell has output of magnitude less than one and, in most cases, we take the zero boundary data.

The equations associated with the interior cell are the same as (3.1). If the Neumann boundary condition is imposed, the equation for the boundary cell at $(k, j),-k<j<k$ is

$$
\begin{aligned}
\frac{d x_{k, j}}{d t}= & -x_{k, j}+z+\left(a_{0,0}+a_{1,0}\right) f\left(x_{k, j}\right)+\left(a_{1,1}+a_{0,1}\right) f\left(x_{k, j+1}\right) \\
& +\left(a_{0,-1}+a_{1,-1}\right) f\left(x_{k, j-1}\right)+a_{-1,1} f\left(x_{k-1, j+1}\right) \\
& +a_{-1,0} f\left(x_{k-1, j}\right)+a_{-1,-1} f\left(x_{k-1, j-1}\right) .
\end{aligned}
$$

In addition, the equation for the corner cell $(k, k)$ is

$$
\begin{aligned}
\frac{d x_{k, k}}{d t}= & -x_{k, k}+z+\left(a_{0,0}+a_{1,0}+a_{0,1}+a_{1,1}\right) f\left(x_{k, k}\right)+\left(a_{0,-1}+a_{1,-1}\right) f\left(x_{k, k-1}\right) \\
& +\left(a_{-1,1}+a_{-1,0}\right) f\left(x_{k-1, k}\right)+a_{-1,-1} f\left(x_{k-1, k-1}\right) .
\end{aligned}
$$

The equations of the other boundary cells and the other corner cells of $T_{k}$ can be analogously obtained. The equations for the other boundary conditions can be obtained in a similar manner.

In the remainder of this presentation, if not in an arithmetic computation, the symbols "+" and "-" are used to represent the positive and negative saturated states 
as well as their output patterns, respectively. Thus, the elements in the set $\mathcal{A}^{\mathbf{Z}^{d}}, \mathcal{A}=$ $\{+,-\}$, give all possible global mosaic patterns. Nevertheless, to save the notations, we shall use $\mathcal{M}^{\infty}=\mathcal{M}^{\infty}(z, A)$ to represent both the sets of feasible global mosaic solutions and patterns corresponding to the parameters $z, A=\left(a_{k, \ell}\right)$. We also denote the set of feasible mosaic solutions (patterns) on $T_{k}$ with boundary condition $B$ by $\mathcal{M}_{k}^{B}=\mathcal{M}_{k}^{B}(z, A)$ and abbreviate it by $\mathcal{M}_{k}$, when no confusion arises.

To obtain patterns on finite lattice, the boundary basic patterns should also be taken into account. However, these boundary basic patterns cannot exist if boundary conditions $N, P, D_{1}$ are imposed, as will be seen later. The following two sections are devoted to deriving the solutions to $Q_{1}$ and $Q_{2}$ for the mosaic patterns of 1-d and 2- $d$ CNN, respectively. The notation $\mathcal{M}_{k}^{B}$ (respectively, $\mathcal{M}^{\infty}$ ) plays the role of $\mathcal{U}_{k}^{B}$ (respectively, $\mathcal{U}$ ) in section 2 and $\pi_{k}$ is the projection on $T_{k}$ now. Conditions $\left(H_{1}\right),\left(H_{2}\right)$ for $\mathrm{CNN}$ are reduced to the following:

$\left(H_{1}^{\prime}\right)$ There is a fixed positive integer $s$ such that for every $u \in \mathcal{M}^{\infty}$ and for each $k>s$ there exists $w^{k} \in X$ with $\pi_{k-s}\left(w^{k}\right)=\pi_{k-s}(u)$ and $\pi_{k}\left(w^{k}\right)$ is a solution (or pattern) of $(\mathrm{CNN})_{k}-B$.

$\left(H_{2}^{\prime}\right)$ Let $x \in \mathcal{M}^{\infty}$ be a global mosaic solution. There is a constant $c_{2}>1 \mathrm{such}$ that for each positive integer $k$ there exists a mosaic solution $v^{k}$ of $(\mathrm{CNN})_{k}-B$ with $\pi_{k-s}\left(v^{k}\right)=\pi_{k-s}(x)$ for some integer $s<(2 k+1) / c_{2}$.

Here, in $\left(H_{2}^{\prime}\right)$, the constant $c_{1}$ in $\left(H_{2}\right)$ is unnecessary, since the mosaic solution $v^{k}$ in $\left(H_{2}^{\prime}\right)$ can always be considered bounded (its components have bounded magnitude). Notably, the condition other than $\left(H_{1}\right)$ in Proposition 2.1 holds. Indeed, $\Gamma\left(\mathcal{M}_{k}^{B}\right) \leq$ $\Gamma_{k}\left(\mathcal{M}^{\infty}\right)$ if $B=N, P, D_{1}, \Gamma\left(\mathcal{M}_{k}^{D_{0}}\right) \leq 2^{4 k-4} \cdot \Gamma_{k-1}\left(\mathcal{M}^{\infty}\right)$ if $d=2$, and $\Gamma\left(\mathcal{M}_{k}^{D_{0}}\right) \leq$ $2^{2} \cdot \Gamma_{k-1}\left(\mathcal{M}^{\infty}\right)$ if $d=1$, according to our approach of forming patterns.

4. CNN on a one-dimensional lattice. The stationary equation of 1- $d$ CNN with the general template $A_{1}=[\alpha, a, \beta]$ is

$$
0=-x_{i}+z+\alpha f\left(x_{i-1}\right)+a f\left(x_{i}\right)+\beta f\left(x_{i+1}\right), \quad i \in \mathbf{Z}^{1} .
$$

If $a \neq 0$, and $x=\left\{x_{i}\right\}_{i=1}^{\infty}$ is a mosaic solution of (4.1), then for each $i \in \mathbf{Z}^{1}$, $(u, v)=\left(x_{i}, y_{i}=f\left(x_{i}\right)\right)$ satisfies

$$
\left\{\begin{array}{l}
v=f(u) \\
v=\frac{1}{a}\left[u-\left(z+\sigma_{1} \alpha+\sigma_{2} \beta\right)\right]
\end{array}\right.
$$

for $\sigma_{1}, \sigma_{2}=1$ or -1 . The expression in $(4.2 \mathrm{~b})$ represents four straight lines on $u-v$ plane if $a, \alpha, \beta \neq 0, \alpha \neq \pm \beta, z$ are given. Herein, these lines are labeled by $L_{\sigma_{1} \sigma_{2}}, \sigma_{1}, \sigma_{2}=1$ or -1 . Notably, on $u-v$ plane, the configuration for the curve of output function in (4.2a) and the four lines in (4.2b) are fixed once $\alpha, \beta, z, a$ are given. For template $A_{1}$ with $\alpha, \beta \neq 0$, there are at most eight basic (mosaic) patterns (refer to Definition 3.3): $\overline{+++}, \overline{++-}, \overline{-++}, \overline{-+-}, \overline{---}, \overline{--+}, \overline{+--}, \overline{+-+}$. We collect them into two groups, $\overline{\mathrm{w}+\mathrm{e}}$ or $\overline{\mathrm{w}-\mathrm{e}}$, where $\mathrm{w}$ (west), e (east) $=$ " + " or "-". If the parameters $\alpha, \beta, z, a$ are given, then the corresponding tentative basic patterns can be determined from (4.2) (the term "tentative" was explained after Definition 3.3). More precisely, if $\alpha, \beta, z, a$ are the parameters with which $L_{\sigma_{1} \sigma_{2}}$ in (4.2b) intersects the piecewise linear curve (4.2a) at $u>1$ (respectively, $u<-1$ ), then $\overline{\mathrm{w}+\mathrm{e}}$ (respectively, $\overline{\mathrm{w}-\mathrm{e}}$ ) is a tentative basic pattern, corresponding to $(\alpha, \beta, z, a)$, where $\mathrm{w}=$ " + ", "-" if $\sigma_{1}=1,-1$, respectively, and e $=$ " + ", "-" if $\sigma_{2}=1,-1$, respectively, as illustrated in Figure 4.1. Therefore, characterizing tentative basic patterns with respect to the parameters $\alpha, \beta, z, a$ amounts to examining how the 
curves in (4.2) intersect. These intersections can actually be classified into finitely many types. This classification then yields a partitioning of the parameter space. In the following, we describe this partitioning along with its associated notations.

The relative position of these four lines depends on the region in Figure 4.2 in which $(\alpha, \beta)$ lies. These relative positions will be used to demonstrate the existence of the basic patterns corresponding to $(z, a)$ in each region of Figure 4.4. This will be explained after we introduce all necessary notations. The notations in Figure 4.2 are of assistance in spelling out the relative position of these four lines. The overhead bar there means "minus sign of " a real number or a symbol. Indeed, these four regions in the half plane $\alpha>\beta$ are described in (4.3):

$$
\begin{aligned}
\Omega_{\alpha \beta} & =\{(\alpha, \beta) \mid \alpha+\beta>\alpha-\beta>-\alpha+\beta>-\alpha-\beta\} ; \\
(\alpha, \beta) \in \Omega_{\alpha \bar{\beta}} & \Leftrightarrow(\alpha,-\beta) \in \Omega_{\alpha \beta}, \\
(\alpha, \beta) \in \Omega_{\bar{\beta} \alpha} & \Leftrightarrow(-\beta, \alpha) \in \Omega_{\alpha \beta}, \\
(\alpha, \beta) \in \Omega_{\bar{\beta} \bar{\alpha}} & \Leftrightarrow(-\beta,-\alpha) \in \Omega_{\alpha \beta} .
\end{aligned}
$$

Notably, $\Omega_{\beta \alpha}$ is symmetric to $\Omega_{\alpha \beta}$ with respect to $\alpha=\beta$ on $\alpha-\beta$ plane. Thus, $y$ is a pattern corresponding to $(z, a)$ with $(\alpha, \beta) \in \Omega_{\beta \alpha}$ if and only if a 180-degree rotation of $y$ is a pattern corresponding to the same $(z, a)$ with $(\alpha, \beta) \in \Omega_{\alpha \beta}$. The same situations hold for the other symmetric regions. Therefore, only $(\alpha, \beta)$ in the half plane $\alpha \geq \beta$ needs to be considered.

There are 25 regions in Figure 4.4, which are denoted by $[1,0 ; 1,0],[m ; 1,0]$, $[1,0 ; n]$, and $[m ; n]$ for $m, n \in I[0,3]$. These notations are interpreted as follows. For example, assume that $(\alpha, \beta) \in \Omega_{\alpha \beta}$, if $(z, a) \in[m ; n]$, the tentative basic patterns are exactly those with at least $(3-m)$ " + " in the nearest neighbors of a positive saturated state and with at least $(3-n)$ "-" in the nearest neighbors of a negative saturated state. If $m=3$ (respectively, $n=3$ ), then it means that there is no restriction for $\mathrm{w}$ and e in $\overline{\mathrm{w}+\mathrm{e}}$ (respectively, $\overline{\mathrm{w}-\mathrm{e}}$ ). If $m=0$ (respectively, $n=0$ ), then any $\overline{\mathrm{w}+\mathrm{e}}$ (respectively, $\overline{\mathrm{w}-\mathrm{e}}$ ) with $\mathrm{w}, \mathrm{e}=$ "+", "-", is not feasible. $(z, a) \in[1,0 ; 1,0]$ indicates that, $\overline{\mathrm{w}+\mathrm{e}}$ with $\mathrm{w}=$ "+" (one "+" for w) and no restriction for e, and $\overline{\mathrm{w}-\mathrm{e}}$ with $\mathrm{w}=$ "- and no restriction for e, are tentative basic patterns. Similar interpretations apply to the notations $[m ; 1,0],[1,0 ; n]$. The following expressions provide the details of these notations. For $\alpha, \beta \in \Omega_{\alpha \beta}$, the notation $\mathcal{B}_{\alpha \beta}([* ; \cdot])$ represents the sets of tentative basic patterns corresponding to each of these parameter region $[* ; \cdot]$ in $z-a$ plane, where "*", "." $=m$ or $n$ or " $1,0 ", m, n \in I[0,3]$. Patterns in each of these sets can be collected into two categories: the ones with center element "+" and the ones with center element "_". Herein, this arrangement is denoted by $\mathcal{B}_{\alpha \beta}([* ; \cdot])=$ $\mathcal{B}_{\alpha \beta}^{+}(*) \cup \mathcal{B}_{\alpha \beta}^{-}(\cdot)$. Similar notations $\mathcal{B}_{\bullet}([* ; \cdot])=\mathcal{B}_{\bullet}^{+}(*) \cup \mathcal{B}_{\bullet}^{-}(\cdot)$ are used for $(\alpha, \beta)$ in the other regions of $\alpha-\beta$ plane. Let us describe these $\mathcal{B}_{\bullet}^{+}(*), \mathcal{B}_{\bullet}^{-}(\cdot)$ now. The following $m, n \in I[1,3]$. For $(\alpha, \beta) \in \Omega_{\alpha \beta}$

$$
\begin{aligned}
\mathcal{B}_{\alpha \beta}^{+}(m) & :=\{\overline{\mathrm{w}+\mathrm{e}} \mid \text { at least }(3-m) \text { of } \mathrm{w} \text { and e are "+" }\}, \\
\mathcal{B}_{\alpha \beta}^{-}(n) & :=\{\overline{\overline{\mathrm{w}-\mathrm{e}}} \mid \text { at least }(3-n) \text { of w and e are "-" }\}, \\
\mathcal{B}_{\alpha \beta}^{+}(1,0) & :=\{\underline{\overline{\mathrm{w}+\mathrm{e}}} \mid \mathrm{w}="+"\}, \\
\mathcal{B}_{\alpha \beta}^{-}(1,0) & :=\{\overline{\overline{\mathrm{w}-\mathrm{e}}} \mid \mathrm{w}=\text { " }-"\} .
\end{aligned}
$$

The overhead bar is used to represent the "negative sign of " symbol "+" or "-" in the following expressions, for example, $\overline{\mathrm{w}}="$ - " if $\mathrm{w}=$ " + ". If $(\alpha, \beta) \in \Omega_{\alpha \bar{\beta}}$, then

$$
\overline{\overline{\mathrm{w}+\mathrm{e}}} \in \mathcal{B}_{\alpha \bar{\beta}}^{+}(m) \Leftrightarrow \underline{\overline{\mathrm{w}+\overline{\mathrm{e}}}} \in \mathcal{B}_{\alpha \beta}^{+}(m),
$$




$$
\begin{aligned}
\overline{\underline{\mathrm{w}-\mathrm{e}}} \in \mathcal{B}_{\alpha \bar{\beta}}(n) \Leftrightarrow \overline{\overline{\mathrm{w}-\overline{\mathrm{e}}}} \in \mathcal{B}_{\alpha \beta}^{-}(n), \\
\mathcal{B}_{\alpha \bar{\beta}}^{+}(1,0)=\overline{\mathcal{B}_{\alpha \beta}^{+}(1,0),} \\
\mathcal{B}_{\alpha \bar{\beta}}^{-}(1,0)=\mathcal{B}_{\alpha \beta}^{-}(1,0) .
\end{aligned}
$$

If $(\alpha, \beta) \in \Omega_{\bar{\beta} \alpha}$, then

$$
\begin{aligned}
& \overline{\overline{\mathrm{w}+\mathrm{e}}} \in \mathcal{B}_{\bar{\beta} \alpha}^{+}(m) \Leftrightarrow \underline{\overline{\mathrm{e}+\mathrm{w}}} \in \mathcal{B}_{\alpha \beta}^{+}(m), \\
& \overline{\overline{\mathrm{w}-\mathrm{e}}} \in \mathcal{B}_{\bar{\beta} \alpha}^{-}(n) \Leftrightarrow \overline{\overline{\mathrm{e}-\mathrm{w}}} \in \mathcal{B}_{\alpha \beta}^{-}(n), \\
& \overline{\underline{\mathrm{w}+\mathrm{e}}} \in \mathcal{B}_{\bar{\beta} \alpha}^{+}(1,0) \Leftrightarrow \mathrm{e}=\text { " }- \text { ", } \\
& \overline{\underline{\mathrm{w}-\mathrm{e}}} \in \mathcal{B}_{\overline{\bar{\beta}} \alpha}^{-}(1,0) \Leftrightarrow \mathrm{e}=\text { " }+" \text {. }
\end{aligned}
$$

If $(\alpha, \beta) \in \Omega_{\bar{\beta} \bar{\alpha}}$, then

$$
\begin{aligned}
& \overline{\underline{\mathrm{w}+\mathrm{e}}} \in \mathcal{B}_{\bar{\beta} \bar{\alpha}}^{+}(m) \Leftrightarrow \underline{\overline{\mathrm{e}+\overline{\mathrm{w}}}} \in \mathcal{B}_{\alpha \beta}^{+}(m), \\
& \overline{\overline{\mathrm{w}-\mathrm{e}}} \in \mathcal{B}_{\overline{\bar{\beta}} \bar{\alpha}}^{-}(n) \Leftrightarrow \underline{\overline{\mathrm{e}-\overline{\mathrm{w}}}} \in \mathcal{B}_{\alpha \beta}^{-}(n), \\
& \mathcal{B}_{\overline{\bar{\beta}} \bar{\alpha}}^{+}(1,0)=\mathcal{B}_{\overline{\bar{\beta}} \alpha}^{+}(1,0), \\
& \mathcal{B}_{\overline{\bar{\beta}} \bar{\alpha}}^{-}(1,0)=\mathcal{B}_{\bar{\beta} \alpha}^{-}(1,0) .
\end{aligned}
$$

In these expressions, w, e could be either "+" or "-" in $\mathcal{B}_{\bullet}^{+}(m)$ (respectively, $\left.\mathcal{B}_{\bullet}^{-}(n)\right)$ if $m=3$ (respectively, $n=3$ ).

As to how the relative position of the slant lines in $(4.2 \mathrm{~b})$ relates to the feasibility of the basic patterns corresponding to $(z, a)$ in each region of Figure 4.4, the following instance is used as an illustration. The first line on the far right side of Figure 4.1 is $L_{1,1}$ if $(\alpha, \beta) \in \Omega_{\alpha \beta}$ and is $L_{1,-1}$ if $(\alpha, \beta) \in \Omega_{\alpha \bar{\beta}}$. The tentative basic pattern corresponding to $(z, a) \in[1 ; 0]$ of Figure 4.4 is $\overline{+++}$ for the first case, and is $\overline{++-}$ for the second case.

Notably, the four lines in (4.2b) degenerate into three lines if $\alpha=\beta$ or $\alpha=-\beta$ with $\alpha, \beta \neq 0$. The tentative basic patterns for $\alpha=\beta>0$ (respectively, $\alpha=\beta<0$, $\alpha=-\beta>0)$ and $(z, a) \in[m ; n]$ in Figure 4.5 are exactly the same for $(\alpha, \beta) \in \Omega_{\alpha \beta}$ (respectively, $\Omega_{\bar{\beta} \bar{\alpha}}, \Omega_{\alpha \bar{\beta}}$ ) and $(z, a) \in[m ; n]$ in Figure 4.4. Furthermore, these four lines in (4.2b) degenerate into two lines if one of $\alpha, \beta$ is zero. The tentative basic patterns for $\alpha>0, \beta=0$ and $(z, a) \in[3 ; 3],[1,0 ; n],[n ; 1,0], n=0,3$ in Figure 4.6 are exactly the same for $(\alpha, \beta) \in \Omega_{\alpha \beta}$ or $\Omega_{\alpha \bar{\beta}}$ and $(z, a) \in[3 ; 3],[1,0 ; n],[n ; 1,0]$ of Figure 4.4, respectively. The same situation holds for $\alpha=0, \beta<0$ and $(\alpha, \beta) \in \Omega_{\bar{\beta} \alpha}$ (or $\Omega_{\bar{\beta} \bar{\alpha}}$ ). The regions $\Omega_{\alpha \beta}, \Omega_{\alpha \bar{\beta}}, \Omega_{\bar{\beta} \alpha}$, and $\Omega_{\bar{\beta} \bar{\alpha}}$, therefore, serve as the representative cases in the $\alpha-\beta$ plane.

The coordinates of these four points B, C, D, E in Figure 4.4 depend on which region $(\alpha, \beta)$ lies in. For example, $\mathrm{B}=1-\alpha-\beta, \mathrm{C}=1-\alpha+\beta, \mathrm{D}=1+\alpha-\beta, \mathrm{E}=$ $1+\alpha+\beta$, if $(\alpha, \beta) \in \Omega_{\alpha \beta}$. The location of $z$-axis in Figure 4.4 (similarly in Figures 4.5 and 4.6) is either below $\mathrm{B}$ or between $\mathrm{B}$ and $\mathrm{C}$ or between $\mathrm{C}$ and $\mathrm{D}$, depending on where $(\alpha, \beta)$ is located in the further partitioned subregions in Figure 4.3.

In association with these partitions of parameter space, we denote by $\mathcal{M}^{\infty}\left([* ; \cdot]_{\alpha, \beta}\right)$, $\mathcal{M}_{k}^{\infty}\left([* ; \cdot]_{\alpha, \beta}\right):=\pi_{k}\left(\mathcal{M}^{\infty}\left([* ; \cdot]_{\alpha, \beta}\right)\right)$, and $\mathcal{M}_{k}^{B}\left([* ; \cdot]_{\alpha, \beta}\right)$, respectively, the set of feasible global mosaic patterns, the set of feasible local mosaic patterns (projected from global patterns) on $T_{k}$, and the set of feasible mosaic patterns on $T_{k}$ with boundary condition $B$, corresponding to parameters $(z, a) \in[* ; \cdot],[* ; \cdot]=[m ; n],[1,0 ; n],[m ; 1,0]$, 


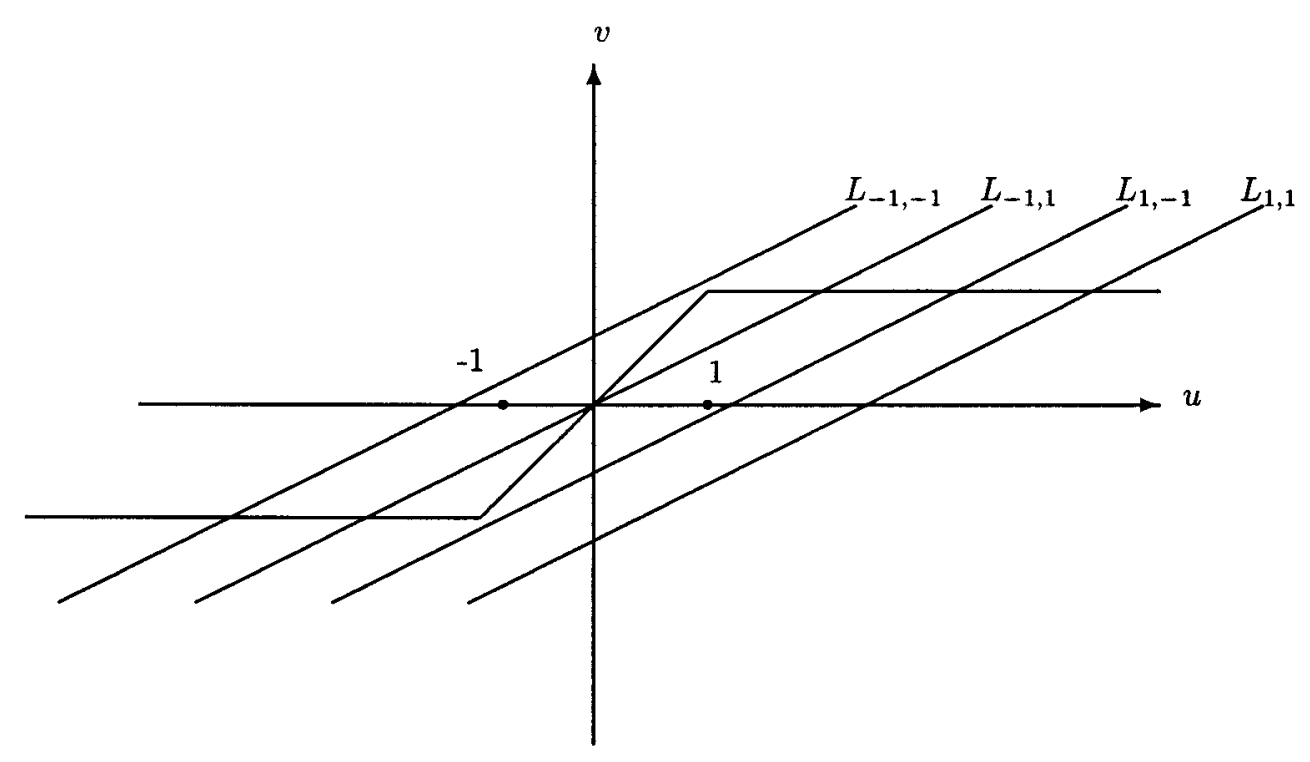

FIG. 4.1. $(\alpha, \beta) \in \Omega_{\alpha \beta}, \quad(z, a) \in[2 ; 1,0]$.

with given $\alpha, \beta$. Occasionally, $\alpha, \beta$ or even $[* ; \cdot]_{\alpha, \beta}$ is omitted in these notations when the parameters have been specified.

We have described the tentative basic patterns which contain the feasible basic patterns corresponding to each parameter region. The global mosaic patterns can be constructed by attaching the feasible basic patterns, as mentioned in section 3 . Therefore, the regime of spatial chaos and pattern formation for the mosaic patterns of CNN on a $1-d$ infinite lattice with template $[\alpha, a, \beta]$ can be completely determined.

TheOREM 4.1. Consider $C N N$ on $\mathbf{Z}^{1}$ with template $[\alpha, a, \beta] . h\left(\mathcal{M}^{\infty}(z, a, \alpha, \beta)\right)>$ 0 if and only if $(z, a, \alpha, \beta)$ lies in one of the following regions:

(i) $(\alpha, \beta) \in \Omega_{\alpha \beta}$ or $\Omega_{\beta \alpha}$ or $\alpha=\beta, \alpha>0$ and $(z, a) \in[m ; n], \quad m, n \geq 2$.

(ii) $(\alpha, \beta) \in \Omega_{\alpha \bar{\beta}}$ or $\Omega_{\beta \bar{\alpha}}$ or $\alpha>0, \beta=0$ or $\beta>0, \alpha=0$ and $(z, a) \in[m ; n]$, $\min \{m, n\} \geq 2, \max \{m, n\}=3$.

(iii) $(\alpha, \beta) \in \Omega_{\bar{\beta} \alpha}$ or $\Omega_{\bar{\alpha} \beta}$ or $\alpha=-\beta$ and $(z, a) \in[m ; n], \min \{m, n\} \geq 2$, $\max \{m, n\}=3$, or $(z, a) \in[3 ; 1,0],[1,0 ; 3]$.

(iv) $(\alpha, \beta) \in \Omega_{\bar{\beta} \bar{\alpha}}$ or $\Omega_{\bar{\alpha} \bar{\beta}}$ or $\alpha=\beta, \alpha<0$ or $\alpha<0, \beta=0$ or $\beta<0, \alpha=0$ and $(z, a) \in[m ; n], \min \{m, n\} \geq 1, \max \{m, n\} \geq 2$, or $(z, a) \in[1,0 ; n],[m ; 1,0]$, $m, n \geq 2$.

The proof of this theorem follows directly from computing the maximum eigenvalue of the corresponding transition matrix in each case. The notion for spatial entropy here is as the topological entropy for Markov shifts. For an account of how the maximal eigenvalue of the transition matrix relates to the spatial entropy of these mosaic patterns, please see [35, p. 341].

Taking the following identification between the indices of a matrix and $1 \times 2$ patterns

$$
1 \leftrightarrow++, \quad 2 \leftrightarrow+-, \quad 3 \leftrightarrow-+, \quad 4 \leftrightarrow--,
$$




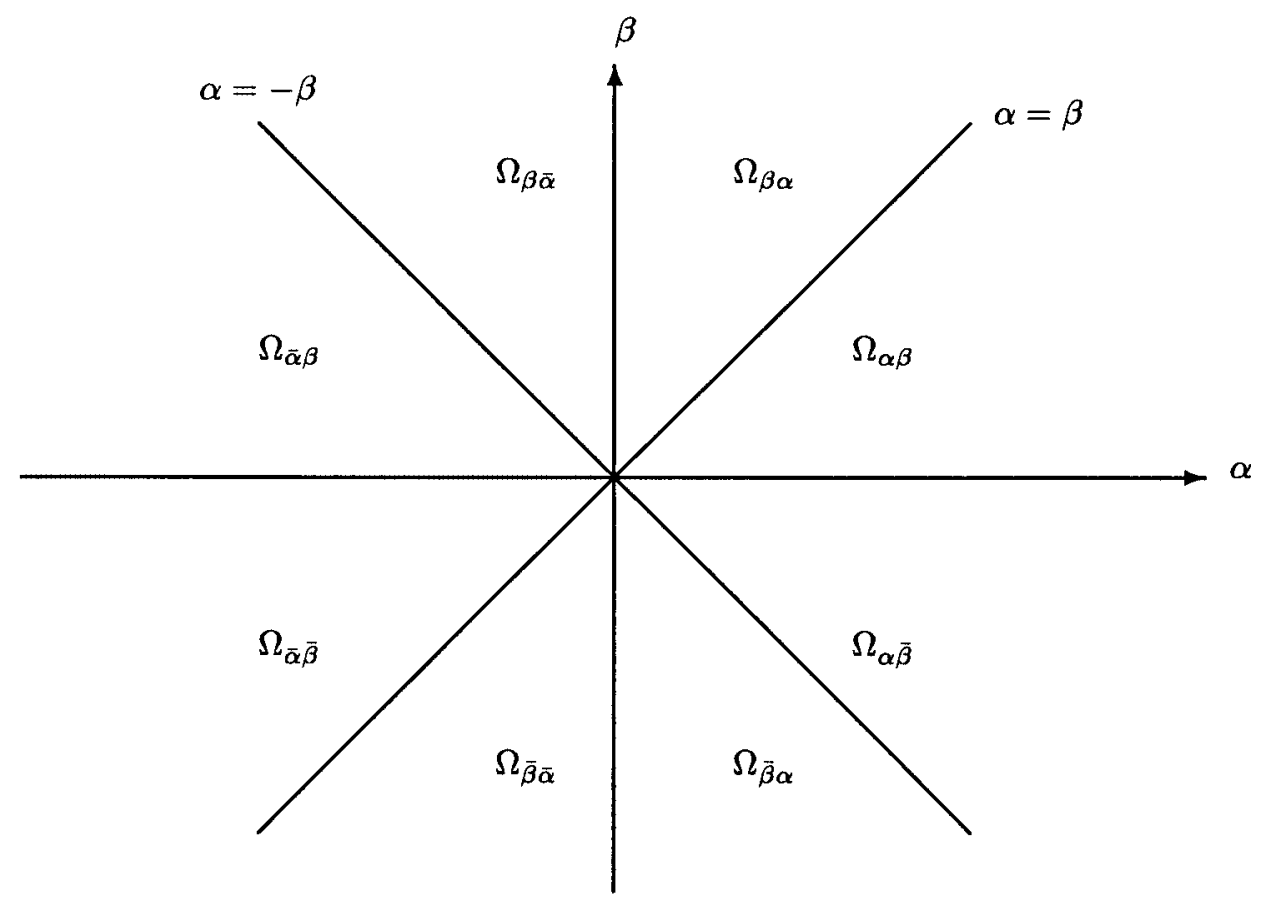

FIG. 4.2. Primary partition of $\alpha-\beta$ plane.

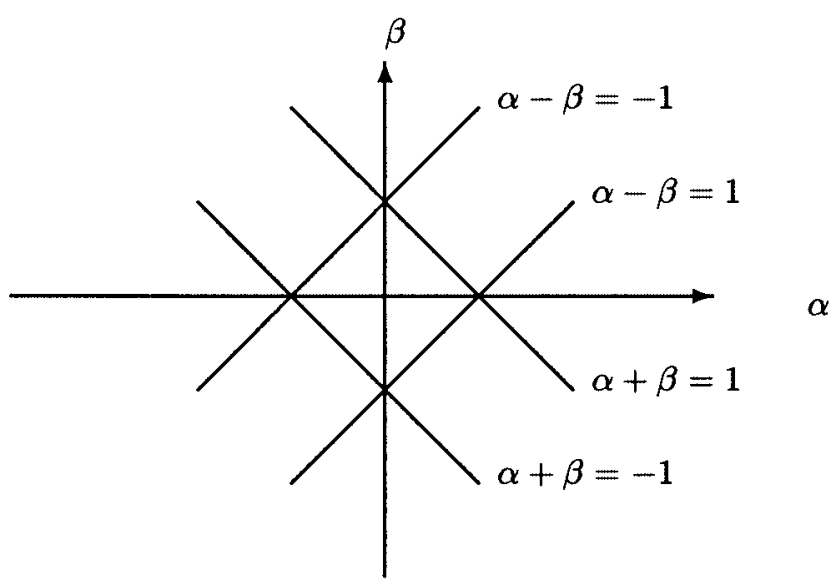

FIG. 4.3. Secondary partition of $\alpha-\beta$ plane.

we consider the transition matrix $M$ in (4.5):

$$
M=M(\mathbf{r}):=\left[\begin{array}{cccc}
r_{1} & r_{2} & 0 & 0 \\
0 & 0 & r_{3} & r_{4} \\
r_{5} & r_{6} & 0 & 0 \\
0 & 0 & r_{7} & r_{8}
\end{array}\right],
$$

where $\mathbf{r}=\left\{r_{j}\right\}_{j=1}^{8}, r_{j}=0$ or $1, j \in I[1,8]$. The formation of mosaic patterns can be described as follows: the $(i, j)$-entry of $M$ is one if and only if the $j$ th $1 \times 2$ pattern 


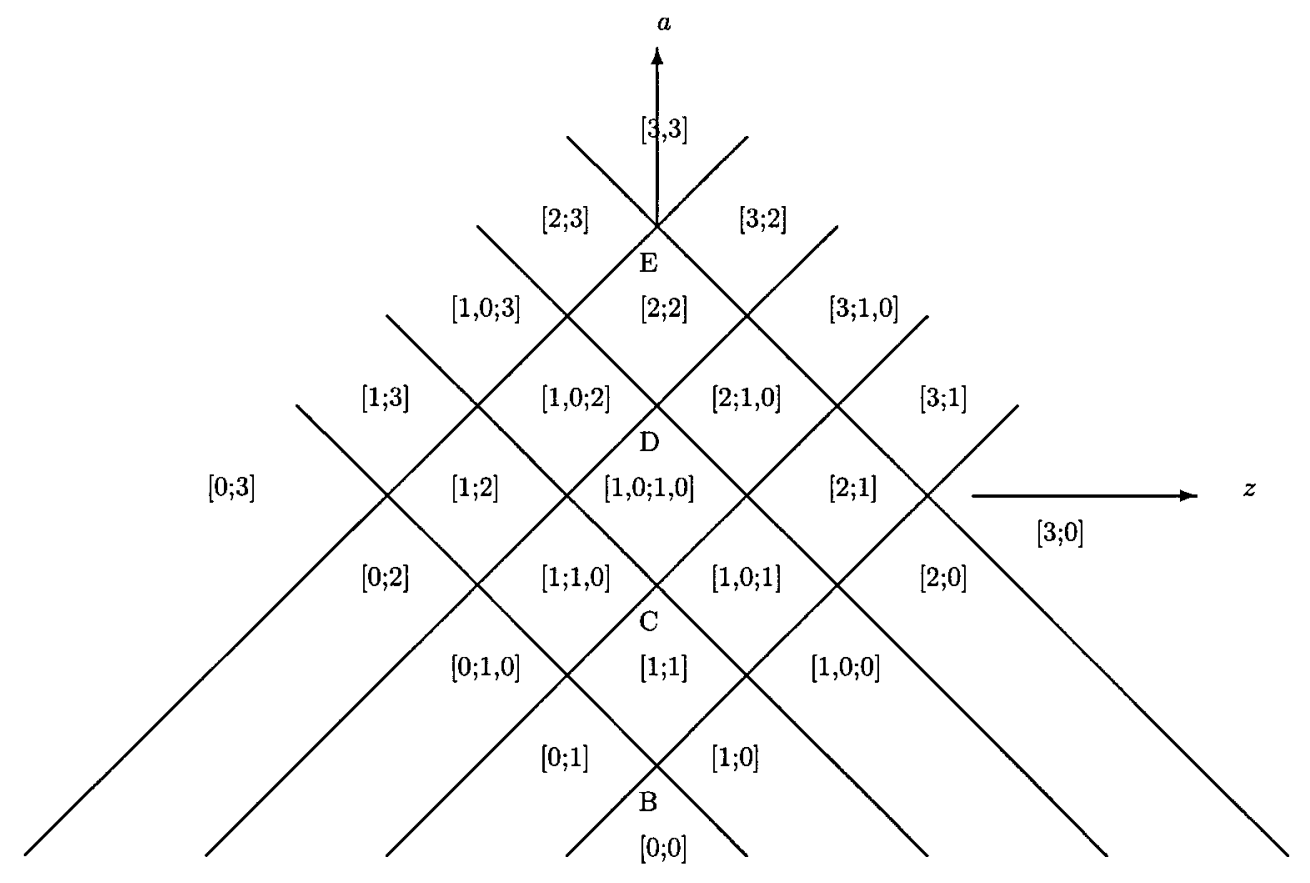

FIG. 4.4. Partition of $z$-a plane for given $\alpha, \beta \neq 0, \alpha \neq \pm \beta$.

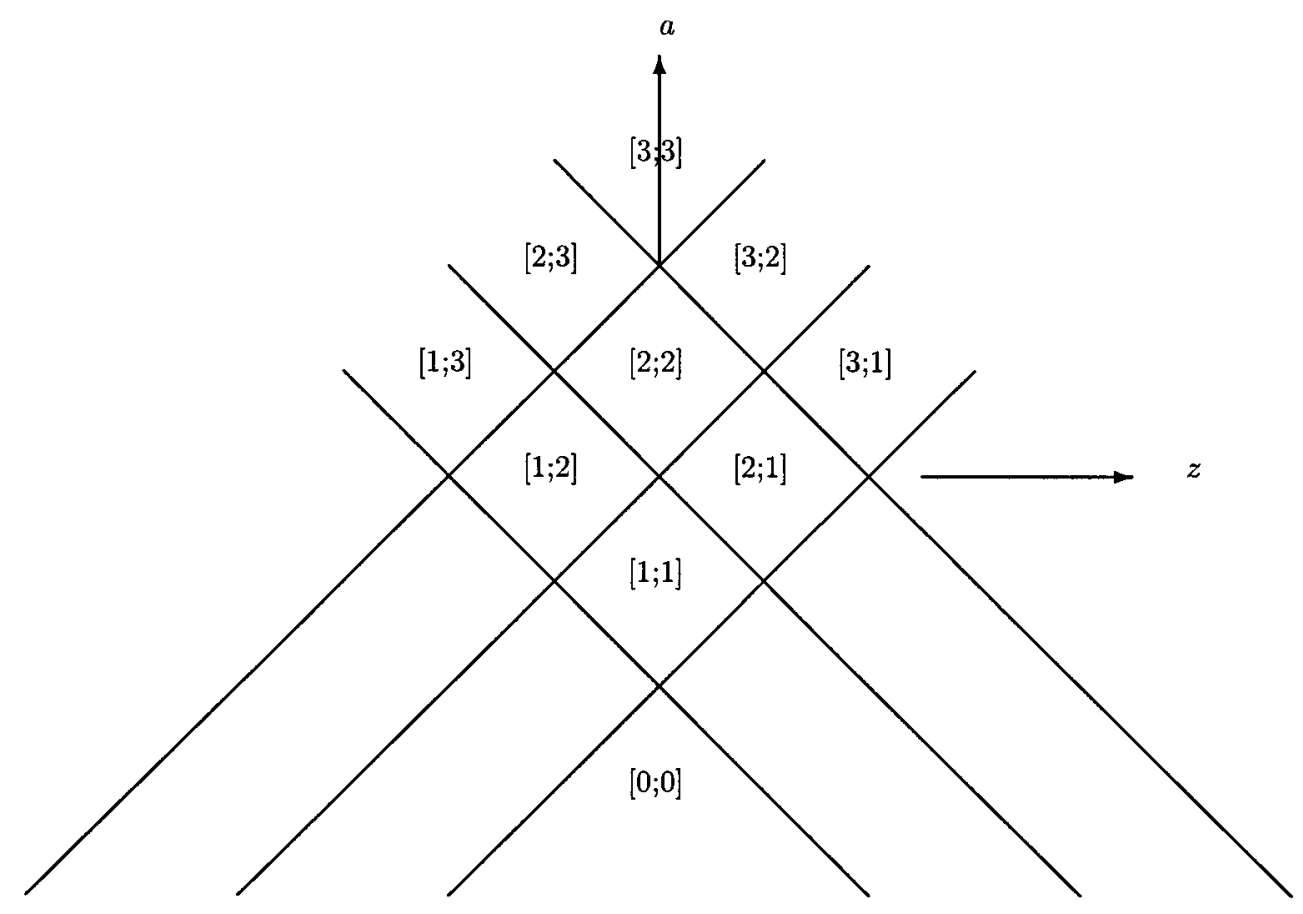

FIG. 4.5. Partition of $z$-a plane for given $\alpha, \beta, \alpha= \pm \beta \neq 0$. 


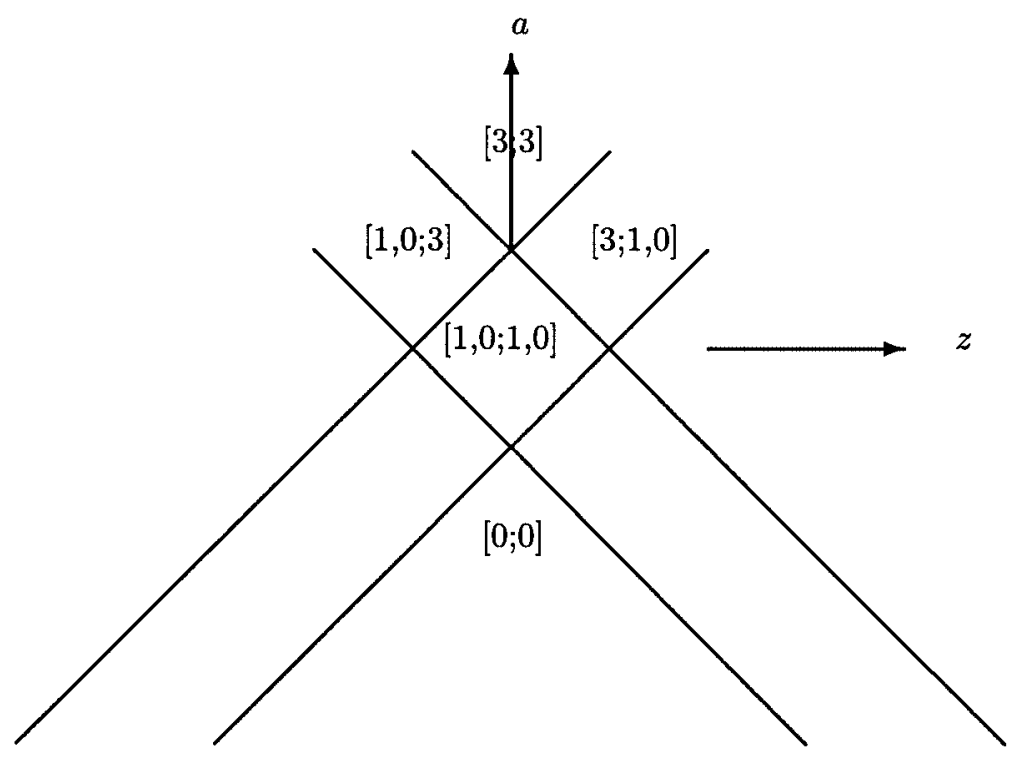

FIG. 4.6. Partition of $z$-a plane for given $\alpha, \beta, \alpha=0$ or $\beta=0$.

in (4.4) can be joined, with one site overlapped, to the right of $i$ th $1 \times 2$ pattern in (4.4) to form a $1 \times 3$ feasible pattern. We collect some properties about the maximum eigenvalue of $M$ in the following lemma, which can be used to verify some cases in Theorem 4.1. Moreover, the motivation of and behind this elementary lemma is to observe how many and what kind of basic mosaic patterns can contribute to positive spatial entropy in this $1-d$ case.

Lemma 4.2. (i) The minimal number of nonzero entries for the matrix $M$ to have eigenvalue greater than 1 is 4 . (ii) The maximal number of nonzero entries for the matrix $M$ to have all eigenvalues no greater than 1 is 6 . (iii) $M$ cannot have more than one eigenvalue greater than 1 for any choice of $r_{j}=0$ or $1, j \in I[1,8]$.

Assume that the parameters are fixed. There corresponds a set of tentative basic patterns (each of them is a $3 \times 1$ pattern) from our previous formulation. In the set, some basic pattern can be attached to itself or other elements in the set, from the left and the right, to form a global pattern. These patterns are the feasible basic (mosaic) patterns, with respect to our Definition 3.3 and its following discussion. On the other hand, some patterns in the set cannot be constructed to form a global pattern by attaching to it any tentative basic patterns. Such a pattern is called a boundary basic pattern since it can only appear near the boundary sites of a finite lattice. Owing to our definition of spatial entropy (without considering boundary conditions), only the feasible basic patterns should be considered. However, it can be seen that, for example, in the following proof (case (iii)) of Theorem 4.1, in constructing the transition matrix, excluding or including these boundary basic patterns does not affect the spatial entropy. That is, the maximal eigenvalues are the same for the corresponding two different transition matrices. One of them is formulated with respect to the tentative basic patterns and the other is for feasible basic patterns only.

To provide more details to the proof of Theorem 4.1, we discuss the critical cases in each item of the theorem as follows. These cases (the following (i)-(iv)) are the 
ones with a minimal amount of feasible basic patterns in each item so that the system exhibits spatial chaos. Herein, we only present the results for the representative regions $\Omega_{\alpha \beta}, \Omega_{\alpha \bar{\beta}}, \Omega_{\bar{\beta} \alpha}$, and $\Omega_{\bar{\beta} \bar{\alpha}}$ on $\alpha-\beta$ plane. In each case, we give the entries of matrix $M$, which can be verified by inspecting the set of feasible basic patterns.

(i) If $(\alpha, \beta) \in \Omega_{\alpha \beta},(z, a) \in[2 ; 2]$, then $r_{3}=r_{6}=0, r_{j}=1, j \in I[1,8], j \neq 3,6$. The set of tentative basic patterns is given in (4.6). Each of its elements is feasible:

$$
\begin{aligned}
& \mathcal{B}_{\alpha \beta}^{+}(2)=\{\overline{\overline{+++}}, \overline{\overline{++}}, \overline{\overline{+++}}\}, \\
& \mathcal{B}_{\alpha \beta}^{-}(2)=\{\overline{\overline{\overline{--}}}, \overline{\overline{\overline{+-+}}}, \overline{\overline{+-}}\} .
\end{aligned}
$$

(ii) If $(\alpha, \beta) \in \Omega_{\alpha \bar{\beta}},(z, a) \in[3 ; 2]$, then $r_{4}=0, r_{j}=1, j \in I[1,8], j \neq 4$. The set of tentative basic patterns is given in (4.7). Each of its elements is feasible:

$$
\begin{aligned}
& \mathcal{B}_{\alpha \bar{\beta}}^{+}(3)=\{\overline{\bullet+\bullet}, \bullet="+" \text { or “-" }\} \\
& \mathcal{B}_{\alpha \bar{\beta}}^{-}(2)=\{\overline{\overline{--+}}, \overline{---}, \underline{\underline{+-+}}\} .
\end{aligned}
$$

(iii) If $(\alpha, \beta) \in \Omega_{\bar{\beta} \alpha},(z, a) \in[3 ; 1,0]$, then $r_{4}=r_{7}=r_{8}=0, r_{j}=1$ otherwise. The set of tentative basic patterns is given in (4.8):

$$
\begin{aligned}
\mathcal{B}_{\bar{\beta} \alpha}^{+}(3) & =\{\overline{\bullet+\bullet}, \bullet="+" \text { or “-" }\}, \\
\mathcal{B}_{\bar{\beta} \alpha}^{-}(1,0) & =\{\overline{\overline{+-+}}, \overline{--+}\} .
\end{aligned}
$$

Note that the basic pattern $\overline{--+}$ in $\mathcal{B}_{\bar{\beta} \alpha}^{-}(1,0)$ is a boundary basic pattern, since no element in the set $\mathcal{B}_{\bar{\beta} \alpha}^{+}(3) \cup \mathcal{B}_{\bar{\beta} \alpha}^{-}(1,0)$ can be attached to the left of it. However, the matrix $M$ with $r_{4}=r_{7}=r_{8}=0, r_{j}=1, j \neq 4,7,8$ has the same maximal eigenvalue as the one with $r_{4}=r_{8}=0, r_{j}=1, j \neq 4,8$.

(iv) If $(\alpha, \beta) \in \Omega_{\bar{\beta} \bar{\alpha}},(z, a) \in[2 ; 1]$, then $r_{1}=r_{4}=r_{7}=r_{8}=0, r_{2}=r_{3}=r_{5}=$ $r_{6}=1$. The set of tentative basic patterns coincides with the one of feasible basic patterns and is given in (4.9):

$$
\begin{aligned}
\mathcal{B}_{\bar{\beta} \bar{\alpha}}^{+}(2) & =\{\overline{\overline{-+-}}, \overline{\overline{++}}, \overline{\overline{-++}}\} \\
\mathcal{B}_{\bar{\beta} \bar{\alpha}}^{-}(1) & =\{\overline{\overline{+-+}}\} .
\end{aligned}
$$

Next, the patterns on a finite lattice are discussed. Notably, the patterns projected onto a finite lattice $T$ from all global mosaic patterns are exactly the same as the set of patterns on $T$ constructed from attaching the feasible basic patterns compatibly. These transition matrices subsequently give us the number of local mosaic patterns on a finite lattice of any size. For example, consider $T_{k}:=\{i \mid-k \leq i \leq k\}$. The number of local mosaic patterns on $T_{k}$ (of size $2 k+1$ ) is

$$
\Gamma_{k}\left(\mathcal{M}^{\infty}\right)=\sum_{i=1}^{4} \sum_{j=1}^{4}\left(M^{2 k-1}\right)_{i, j} .
$$

While attempting to describe patterns on a finite lattice, the transition matrix should be formulated with consideration of both the feasible basic patterns and the boundary basic patterns. However, it is not difficult to verify that if boundary condition $N$ or $P$, or $D_{1}$ is imposed, the boundary basic patterns fail to exist.

In fact, the notion of transition matrix that describes the pattern forming tells us more. The $(i, j)$-entry of $M^{2 k-1}$ gives the number of feasible mosaic patterns on 
$T_{k}$ with $i$ th $1 \times 2$ pattern in (4.4) at the two sites to the far left of $T_{k}$ and $j$ th $1 \times 2$ pattern in (4.4) at the two sites to the far right of $T_{k}$. For instance, the $(1,2)$-entry of $M^{2 k-1}$ gives the number of mosaic patterns on $T_{k}$ with the left-hand side having " ++ " and the right-hand side having " +- ", that is, patterns of the form in (4.10):

$$
++\cdots \cdots+\cdot \text {. }
$$

Using this information and our formulation of basic mosaic patterns allow us to count the number of mosaic patterns on $T_{k}$ with any boundary conditions. $Q_{1}$ can then be answered completely for the mosaic patterns of CNN. $Q_{2}$ can also be resolved completely for the mosaic patterns of $\mathrm{CNN}$, with our formulation of basic mosaic patterns.

Next, the mosaic patterns on $T_{k}$, which satisfy various boundary conditions are considered. Since the pattern forming described by transition matrix works only in a 1-d lattice in general, we attempt to derive the solution for $Q_{1}$ via the setting of transition matrix as well as Proposition 2.1. The latter gives us experience in treating the problems in two and higher-dimensional lattices. Notably, the condition other than $\left(H_{1}^{\prime}\right)$ in Proposition 2.1 has been verified at the end of section 3. In deriving the solution for $Q_{2}$, basically, we verify if $\left(H_{2}^{\prime}\right)$ in section 3 is satisfied. If a boundary reconstruction scheme can be developed for every local pattern projected from a global pattern onto $T_{k}$ (abbreviated as local pattern or projected pattern), for given parameters, then $Q_{2}$ is true for these parameters. In the 1- $d$ case, if this boundary reconstruction scheme cannot be obtained for some projected pattern, then at the same time, $Q_{2}$ cannot be true. Though $\left(H_{2}^{\prime}\right)$ and $Q_{2}$ are discussed in the context of stationary solutions, it is more convenient to manipulate the boundary reconstruction in terms of the output patterns which correspond to the stationary solutions. Our discussions are divided into four parts (I), (II), (III), (IV). They correspond to $(\alpha, \beta) \in \Omega_{\alpha \beta}, \Omega_{\alpha \bar{\beta}}, \Omega_{\bar{\beta} \alpha}, \Omega_{\bar{\beta} \bar{\alpha}}$, respectively. Herein, only the typical and representative cases in each item are presented.

Notably, if $(z, a) \in[3 ; 3]$, for any given $\alpha, \beta$, formation of patterns is unaffected by any boundary condition. Moreover, if $h\left(\mathcal{M}^{\infty}\right)=0$ in some parameter region, then $h_{B}=0$ for boundary condition $B=N, P, D_{1}, D_{0}$.

(I) For $(\alpha, \beta) \in \Omega_{\alpha \beta}$, Neumann B.C. is very natural in the sense that $\pi_{k}(x)$ is a solution of $(\mathrm{CNN})_{k}-N$ for every mosaic solution $x$ of $(\mathrm{CNN})_{\infty}$. Therefore, $Q_{1}, Q_{2}$ are true for Neumann B.C. and any $(z, a)$; see Theorem 4.3 and Table 4.1 in Theorem 4.4 .

Consider $(z, a) \in[2 ; 2]$. Since periodic boundary condition requires coincidence of the patterns at the sites to the far right and the ones to the far left, some projected patterns $\pi_{k}(y)$ (solutions $\left.\pi_{k}(x)\right)$ certainly fail to be feasible patterns of $(\mathrm{CNN})_{k}-P$. For example, patterns $\pi_{k}(y)$ of the form in (4.11):

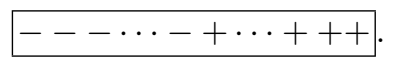

Also, imposing certain prescribed boundary data obviously affects the formation of patterns. For instance, imposing positive saturated state at the left end, then any pattern of the form enclosed in (4.12) cannot exist:

$$
+-++\cdots \cdots \cdots \cdots \cdot \text {. }
$$

Furthermore, if $a<1+\beta$, imposing zero data on the left end, the pattern enclosed in (4.13) cannot exist:

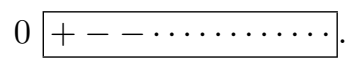


On the other hand, given any mosaic pattern $y$ of $(\mathrm{CNN})_{\infty},\left(\pi_{k}(y)\right)_{i}, i= \pm k, \pm(k-1)$, $\pm(k-2)$, can always be amended so that they become $\left(\pi_{k}(y)\right)_{i}=1$. That is, the outputs near the two ends of any local pattern $\pi_{k}(y), y=\left\{f\left(x_{i}\right)\right\}$, can be changed, without losing the feasibility, into the one in the form (4.14):

$$
\mathrm{w}+++\cdots \cdots+++ \text { e. }
$$

A pattern of this form certainly satisfies the periodic B.C. and Dirichlet B.C., with any prescribed boundary values $\mathrm{w}$ and e imposed on the left and the right ends. Thus, $\left(H_{1}^{\prime}\right)$ (see section 3) is satisfied, and $h=h_{N}=h_{P}=h_{D_{1}}=h_{D_{0}}$ can be verified, by Proposition 2.1. $Q_{2}$ is also true for all these boundary conditions, by Proposition 2.2. (4.15):

If $(z, a) \in[3 ; 1,0]$, the set of projected patterns $\pi_{k}\left(\mathcal{M}^{\infty}\right)$ consists of elements in

$$
\{---\cdots-+\cdots+++, \quad---\cdots---,+++\cdots+++\} .
$$

In this set, patterns of the form (4.11) do not and cannot be amended to satisfy the periodic boundary conditions, so $Q_{2}$ is false. If positive saturated state at the left end is imposed, the "-" strip pattern in (4.15) fails to exist. If negative saturated states are imposed at both ends, then $\mathcal{M}_{k}^{D_{1}}=\pi_{k}\left(\mathcal{M}^{\infty}\right)$. Thus, $Q_{2}$ can be true or false in this case, depending on different prescribed data. The other results in Theorem 4.4 can be similarly obtained.

(II) For $(\alpha, \beta) \in \Omega_{\alpha \bar{\beta}},(z, a) \in[3 ; 2]$. Imposing Neumann B.C. forces a projected pattern on $T_{k}$ of the form in (4.16) fail to exist:

$$
\cdots \cdots \cdots \cdots++- \text {. }
$$

However, the outputs near the right edge can be changed, without losing the feasibility. In doing so, the pattern becomes the form in (4.17), which satisfies the Neumann B.C.:

$$
\cdots \cdots \cdots \cdot \cdots-++ \text {. }
$$

Thus, $Q_{1}, Q_{2}$ are true for Neumann B.C., by Propositions 2.1 and 2.2. It is more complicated for periodic boundary conditions. Here, the setting of transition matrix is adopted. Many (actually, the number grows exponentially in the size of the lattice) patterns on $T_{k}$ do not satisfy periodic B.C. However, the spatial entropy remains unchanged if we only count the patterns that satisfy periodic B.C. This can be verified by computing the transition matrix to the power $2 k-1$, that is, $M^{2 k-1}$. As mentioned earlier

$$
\Gamma_{k}\left(\mathcal{M}^{\infty}([3 ; 2])\right)=\sum_{i=1}^{4} \sum_{j=1}^{4}\left(M^{2 k-1}\right)_{i, j},
$$

where $M$ is given in the proof (ii) of Theorem 4.1. If periodic boundary condition is demanded, then the number of mosaic patterns on $T_{k}$ is $\Gamma\left(\mathcal{M}_{k}^{P}([3 ; 2])\right)=$ $\Gamma_{k}\left(\mathcal{M}^{\infty}([3 ; 2])\right)-\left(M^{2 k-1}\right)_{4,2}$. This number can be estimated or actually computed so that $h=h_{P}$ can be verified. However, $Q_{2}$ is false because some projected patterns do not and cannot be amended to satisfy the periodic boundary conditions. For Dirichlet B.C., if some positive saturated state or the zero boundary data (with parameters satisfying $z-a \geq \beta-1$ ) at the left end is imposed, then the local patterns on $T_{k}$ of the forms in (4.18) cannot exist:

$$
\begin{array}{ll}
+--+\cdots \cdots, & +---\cdots \cdots, \\
0--+\cdots \cdots, & 0---\cdots \cdots .
\end{array}
$$


It even forces the basic patterns $\overline{--+}, \overline{\overline{--}}$ not to exist in the interior of $T_{k}$. Restated, if certain $D_{0}$ or $D_{1}$ is considered, the set of feasible basic patterns becomes $\{\overline{\boldsymbol{\bullet + \bullet}}, \bullet="+$ " or " $-"\} \cup\{\overline{\overline{+-+}}\}$. Thus, $Q_{2}$ is obviously false in this case. For some other prescribed boundary values, it is possible that $\mathcal{M}_{k}^{D_{1}}=\mathcal{M}_{k}^{D_{0}}=\pi_{k}\left(\mathcal{M}^{\infty}\right)$. Therefore, $Q_{2}$ could be true or false. Nevertheless, the transition matrix associated with these basic patterns is $M=M(\mathbf{r})$ with $r_{1}=r_{2}=r_{3}=r_{5}=r_{6}=1, r_{j}=0$ for the other $j$. Moreover, the maximal eigenvalue of this transition matrix is the same as the one given in the proof (ii) of Theorem 4.1. Therefore, the spatial entropy still remains unchanged. That is, $h=h_{D_{0}}=h_{D_{1}}$.

If $(z, a) \in[2 ; 2]$, local patterns of the form in (4.19) do not and cannot be modified to satisfy the Neumann B.C. $Q_{2}$ is thus false for $(\mathrm{CNN})_{k}-N$ :

$$
\cdots \cdots++-+-+, \quad \cdots \cdots+-+-+- \text {. }
$$

We omit the other cases.

(III) If $(\alpha, \beta) \in \Omega_{\bar{\beta} \alpha}$, the phenomena is similar to the ones in $(\alpha, \beta) \in \Omega_{\alpha \bar{\beta}}$ and is omitted.

(IV) If $(\alpha, \beta) \in \Omega_{\bar{\beta} \bar{\alpha}},(z, a) \in[2 ; 1]$, local patterns of the form in (4.20) exist for any B.C.,

$$
+-\cdots \cdots \cdots++ \text {. }
$$

Actually, if the Neumann B.C. is imposed, a projected pattern $\pi_{k}(y)$ on $T_{k}$ is feasible only if $\left(\pi_{k}(y)\right)_{ \pm k}=1,\left(\pi_{k}(y)\right)_{ \pm(k-1)}=-1$, that is, a pattern of the form (4.20). Restated, the number of feasible mosaic patterns on $T_{k}$ is $\left(M^{2 k-1}\right)_{2,3}$, where $M$ is given in the proof (iv) of Theorem 4.1. It can be computed that, still, $h=h_{N}$. The discussions on other boundary conditions resemble the preceding ones and are, therefore, omitted. We have the following theorems which resolve $Q_{1}$ and $Q_{2}$ respectively, for mosaic patterns of CNN on a 1-d lattice.

THEOREM 4.3. Consider a 1-d CNN with template $A_{1}=[\alpha, a, \beta], h=h_{N}=$ $h_{P}=h_{D_{1}}=h_{D_{0}}$.

TheOrEm 4.4. Consider a 1-d CNN with template $A_{1}=[\alpha, a, \beta]$. The solutions for $Q_{2}$ are given in the following Tables 4.1-4.4 for each region of Figure 4.2 in the range $\alpha \geq \beta$. In the tables, " $T$ " denotes true, "F" represents false, and "TF" refers to true or false, depending on what values of boundary data are prescribed or on different parameters in the same region.

For the parameters $(z, a) \in[3 ; 3]$ with any $\alpha, \beta$, no restriction is placed on the states (patterns) in the nearest neighbors of a "+" or a "-" (w, e can even be defect states in $\overline{\mathrm{w}+\mathrm{e}}, \overline{\mathrm{w}-\mathrm{e}}$ ). This is confirmed from the previous description of feasible basic patterns as well as from verifying $x_{i}>1$ (respectively, $x_{i}<-1$ ) if the output at the site $i$ is "+" (respectively, "-") in (4.1), for $(z, a) \in[3 ; 3]$. Thus, if $(z, a) \in[3 ; 3]$, $\pi_{k}(x)$ is feasible on $T_{k}$ with boundary condition $B=N, P, D_{1}, D_{0}$, for every mosaic solution $x$ of $(\mathrm{CNN})_{\infty}$. The case $B=P$ requires further explanation. Consider $u=\left\{u_{j}\right\}_{j \in T_{k}}, u_{j} \in \mathbf{R}$. In our formulation of periodic boundary condition, the coincidence $u_{-k}=u_{k}$ yields a circular array of length $2 k$, since the size of $T_{k}$ is $2 k+1$. There is another formulation: attach (without overlapping) $u_{-k}$ to $u_{k}$ and form a feasible pattern on a $2 k+1$ circular array. This formulation does not change the answer to $Q_{2}$ for $(\mathrm{CNN})_{k}-P$ in each case, as the norm $\|\cdot\|_{\ell_{q}^{2}}, q>1$ is adopted. When using $\|\cdot\|_{\ell^{\infty}}$ norm, we choose this nonoverlapping formulation. In addition, it follows from the preceding discussion (I) for $(\alpha, \beta) \in \Omega_{\alpha \beta}$, that $\pi_{k}(x)$ is a solution of 
TABLE 4.1

$(\alpha, \beta) \in \Omega_{\alpha \beta}$ or $\alpha=\beta>0$.

\begin{tabular}{|c|c|c|c|c|}
\hline Boundary condition & Neumann & Periodic & Dirichlet $D_{1}$ & Dirichlet $D_{0}$ \\
\cline { 1 - 4 }$(z, a) \in$ & & & & \\
\hline$[3 ; 3],[3 ; 2],[2 ; 3]$ & $\mathrm{T}$ & $\mathrm{T}$ & $\mathrm{T}$ & $\mathrm{T}$ \\
\hline$[3 ; 1,0],[1,0 ; 3]$ & $\mathrm{T}$ & $\mathrm{F}$ & $\mathrm{TF}$ & $\mathrm{TF}$ \\
{$[2 ; 1,0],[1,0 ; 2]$} & & & & $\mathrm{TF}$ \\
\hline$[2 ; 1],[1 ; 2]$ & $\mathrm{T}$ & $\mathrm{T}$ & $\mathrm{TF}$ & $\mathrm{TF}$ \\
\hline$[1,0 ; 1,0],[1,0 ; 1],[1 ; 1,0]$ & $\mathrm{T}$ & $\mathrm{T}$ & $\mathrm{F}$ & \multicolumn{2}{|r}{} \\
\hline
\end{tabular}

TABLE 4.2

$(\alpha, \beta) \in \Omega_{\alpha \bar{\beta}}$ or $\beta=0, \alpha>0$.

\begin{tabular}{|c|c|c|c|c|}
\hline Boundary condition & \multirow[t]{2}{*}{ Neumann } & \multirow[t]{2}{*}{ Periodic } & \multirow[t]{2}{*}{ Dirichlet $D_{1}$} & \multirow[t]{2}{*}{ Dirichlet $D_{\mathrm{C}}$} \\
\hline$(z, a) \in$ & & & & \\
\hline$[3 ; 3]$ & $\mathrm{T}$ & $\mathrm{T}$ & $\mathrm{T}$ & $\mathrm{T}$ \\
\hline $\begin{array}{c}{[3 ; 2],[2 ; 3]} \\
{[3 ; 1,0],[1,0 ; 3]}\end{array}$ & $\mathrm{T}$ & $\mathrm{F}$ & $\mathrm{TF}$ & $\mathrm{TF}$ \\
\hline$[2 ; 2]$ & $\mathrm{F}$ & $\mathrm{F}$ & $\mathrm{F}$ & $\mathrm{TF}$ \\
\hline$[2 ; 1,0],[1,0 ; 2],[1,0 ; 1,0]$ & $\mathrm{T}$ & $\mathrm{T}$ & $\mathrm{F}$ & $\mathrm{TF}$ \\
\hline$[1,0 ; 1],[1 ; 1,0]$ & $\mathrm{T}$ & $\mathrm{T}$ & $\mathrm{TF}$ & $\mathrm{TF}$ \\
\hline
\end{tabular}

TABLE 4.3

$(\alpha, \beta) \in \Omega_{\bar{\beta} \alpha}$ or $\alpha=-\beta$.

\begin{tabular}{|c|c|c|c|c|}
\hline Boundary condition & Neumann & Periodic & Dirichlet $D_{1}$ & Dirichlet $D_{0}$ \\
\cline { 1 - 4 }$(z, a) \in$ & & & & $\mathrm{T}$ \\
\hline$[3 ; 3]$ & $\mathrm{T}$ & $\mathrm{T}$ & $\mathrm{T}$ & $\mathrm{TF}$ \\
\hline$[3 ; 2],[2 ; 3]$ & $\mathrm{T}$ & $\mathrm{F}$ & $\mathrm{TF}$ & $\mathrm{T}$ \\
\hline$[3 ; 1,0],[1,0 ; 3]$ & $\mathrm{T}$ & $\mathrm{T}$ & $\mathrm{T}$ & $\mathrm{TF}$ \\
\hline$[2 ; 2],[1,0 ; 1,0]$ & $\mathrm{F}$ & $\mathrm{F}$ & $\mathrm{F}$ & \\
\hline$[2 ; 1,0],[1,0 ; 2]$ & & & &
\end{tabular}

TABLE 4.4

$(\alpha, \beta) \in \Omega_{\bar{\beta} \bar{\alpha}}$ or $\alpha=0, \beta<0$ or $\alpha=\beta<0$.

\begin{tabular}{|c|c|c|c|c|}
\hline Boundary condition & Neumann & Periodic & Dirichlet $D_{1}$ & Dirichlet $D_{0}$ \\
\cline { 1 - 2 }$(z, a) \in$ & & & & \\
\hline$[3 ; 3],[3 ; 2],[2 ; 3]$ & & $\mathrm{T}$ & $\mathrm{T}$ & $\mathrm{T}$ \\
{$[2 ; 1,0],[1,0 ; 2]$} & $\mathrm{T}$ & & & $\mathrm{TF}$ \\
\hline$[2 ; 1],[1 ; 2],[2 ; 2]$ & & $\mathrm{F}$ & $\mathrm{F}$ & $\mathrm{T}$ \\
\hline
\end{tabular}

$(\mathrm{CNN})_{k}-N$ for every mosaic solution $x$ of $(\mathrm{CNN})_{\infty}$. Hereinafter, we have the following results in the phase space $X=\ell^{\infty}$.

Theorem 4.5. Assume that $X=\ell^{\infty}$.

(i) Let $\mathcal{U}=\mathcal{M}^{\infty}([3 ; 3])$, the set of global mosaic patterns with $(z, a) \in[3 ; 3]$ for any $\alpha, \beta . Q_{2}$ is true for $(\mathrm{CNN})_{k}-B, B=N, P, D_{1}, D_{0}$.

(ii) Let $\mathcal{U}=\mathcal{M}^{\infty}$, the set of all global mosaic patterns corresponding to any $(z, a)$, $\alpha, \beta>0 . Q_{2}$ is true for $(\mathrm{CNN})_{k}-N$. 
5. CNN on two-dimensional lattice. Consider a family of two-parametered templates,

$$
\left[\begin{array}{ccc}
0 & \eta_{4} \beta & 0 \\
\eta_{1} \beta & a & \eta_{3} \beta \\
0 & \eta_{2} \beta & 0
\end{array}\right]
$$

where $\eta_{j}=1$ or $-1, j \in I[1,4]$. These templates are square-cross (their corner entries are zeros). We denote by $S A, H A$, and $N A$, respectively, the symmetric template if $\eta_{j}=1, j \in I[1,4]$, horizontally symmetric template if $\eta_{j}=1, j=1,3,4, \eta_{2}=-1$, and nonsymmetric template if $\eta_{3}, \eta_{4}=1, \eta_{1}, \eta_{2}=-1$. Pattern formation and spatial chaos for CNN have been studied by Juang and Lin [29] for $S A$-template and by Shih [37] for $H A$ - and $N A$-templates. The analysis there can be extended to any templates of the form (5.1) and to analogous diagonal-cross templates.

The parameter space can be partitioned by the same methodology as in the 1- $d$ case in section 4. Let us illustrate the one for $S A$-template. Assume that $a \neq 0$. If $x=\left\{x_{i, j}\right\}$ is a global mosaic solution of (3.1) with template $S A$, then for each $(i, j) \in \mathbf{Z}^{2},(u, v)=\left(x_{i, j}, y_{i, j}\right)$ satisfies

$$
\left\{\begin{array}{l}
v=f(u) \\
v=\frac{1}{a}\{u-(z+2 k \beta)\}
\end{array}\right.
$$

for some $k \in I[-2,2]$. Given $\beta \neq 0, a \neq 0$ and $z$, on $u$ - $v$ plane, there are five straight lines $L_{k}$ indexed by $k \in I[-2,2]$ in (5.3). In each line $1 / a$ is the slope and $(z+2 k \beta)$ is the $u$-intercept. The number of lines, among these five, that intersect (5.2) at $u>1$, and $u<-1$ determines, respectively, the number $m$, and $n$ in $[m ; n]$ of Figure 5.1. More explicitly, assume that $\beta>0$ is fixed and $p, q \in I[-2,2]$. Let $a, z \in \mathbf{R}$ be such that $L_{k}, k \in I[p, 2]$ are the only $(3-p)$ lines that intersect (5.2) at $u>1$ and $L_{k}$, $k \in I[-2, q]$, are the only $(q+3)$ lines that intersect $(5.2)$ at $u<-1$. We collect these $a$ and $z$ and form the set $[m ; n]\left(=[m ; n]_{\beta}\right)$, where $m=3-p, n=q+3$. The case $\beta<0$ can be explained in a similar manner. These $[m ; n], m, n \in I[1,5]$ are depicted in Figure 5.1 for $\beta>0$. The characterization of tentative basic mosaic patterns with respect to the parameters are given as follows. Let e, w, s, n represent "+" or "-". A basic mosaic pattern for square-cross template is of the form

$$
\begin{array}{ccccc}
\mathrm{n} & & \mathrm{n} & \\
+ & \mathrm{e} & \mathrm{w} & - & \mathrm{e} \\
\mathrm{s} & & & \mathrm{s} &
\end{array} .
$$

If $(z, a) \in[m ; n], \beta>0$ (respectively, $\beta<0$ ), then the corresponding tentative basic patterns with "+" at the center, that is, of the form in (5.4a), are those having at least $(5-m)$ " + " (respectively, "-") in $\{\mathrm{e}, \mathrm{w}, \mathrm{s}, \mathrm{n}\}$; and the corresponding tentative basic patterns with "-" at the center, that is, of the form in $(5.4 \mathrm{~b})$, are those with at least $(5-n)$ "-" (respectively, "+") in $\{\mathrm{e}, \mathrm{w}, \mathrm{s}, \mathrm{n}\}$. If $m=5$ (respectively, $n=5$ ), then each of e, w, s, n could be "-" or " +" in the tentative basic patterns with "+" (respectively, "-") at the center.

In this section, we study how boundary conditions affect pattern formation and spatial chaos for CNN with the templates $S A$ and $H A$. It has been shown that CNN with $S A$-template exhibits spatial chaos if and only if $\min \{m, n\} \geq 2$ and 
$\max \{m, n\} \geq 3$ if $\beta>0$ and $\min \{m, n\} \geq 2$ and $\max \{m, n\} \geq 3$ or $[m ; n]=$ $[5 ; 1],[1 ; 5] ;[4 ; 1],[1 ; 4]$ if $\beta<0$; see [29]. In general, if $m, n$ are larger, then the formation for mosaic patterns is less restrictive and hence there are more patterns. On the one hand, adjusting the states (or outputs) at certain sites without losing the feasibility seems to be more possible. On the other hand, there are also more patterns to be considered in constructing a boundary reconstruction scheme in verifying conditions $\left(H_{1}^{\prime}\right)$ and $\left(H_{2}^{\prime}\right)$. The results in deriving the solutions to $Q_{1}, Q_{2}$ for mosaic patterns of CNN with $S A$-template are given in Theorem 5.3 and Theorem 5.4. Some counterexample to $h=h_{D}$ is given following Theorem 5.4. We also give a counterexample to $h=h_{N}$ using template $H A$, as discussed in Example 2.

Since the template in (5.1) is square-cross, the boundary sites in this case become (without corners outside $T_{k}$ )

$$
\mathbf{b}:=\{(-k-1, j),(k+1, j),(i,-k-1),(i, k+1),-k \leq i \leq k,-k \leq j \leq k\} .
$$

The following notion of total output is effective in justifying the existence and nonexistence of patterns.

Definition 5.1. For template $A=S A$ or $H A$ or $N A$, the signed total output at the site $(i, j)$ is defined as

$$
T O(i, j, \bullet)=f\left(x_{i+1, j}\right)+f\left(x_{i, j+1}\right)+\eta_{1} f\left(x_{i-1, j}\right)+\eta_{2} f\left(x_{i, j-1}\right),
$$

where $\left(\eta_{1}, \eta_{2}\right)$ is as in (5.1).

Herein, " $\bullet=+$, or - , or $\times$; it represents positive mosaic, or negative mosaic, or defect pattern at the site $(i, j) . T O(i, j, \bullet)$ is abbreviated as $T O(+), T O(-)$, or $T O(\times)$, when there is no confusion. The following lemma resembles Lemma 5.1 in [37]. It is used to justify the coexistence of "+" and "-" on a basic set. Its proof follows from direct computations on the equations (3.1).

Lemma 5.2. Let $x=\left\{x_{i, j}\right\}$ be a global mosaic solution of (3.1) with template (5.1).

(i) $x_{i, j}>1$ if and only if $T O(i, j ;+)>(1-a-z) / \beta, \beta>0$ or $T O(i, j ;+)<$ $(1-a-z) / \beta, \beta<0$.

(ii) $x_{i, j}<-1$ if and only if $T O(i, j ;-)<(a-1-z) / \beta, \beta>0$ or $T O(i, j ;-)>$ $(a-1-z) / \beta, \beta<0$.

ThEOREM 5.3. Consider the CNN with template $S A$ and $a, \beta \neq 0$. The questions $Q_{1}, Q_{2}$ for the set of mosaic solutions (patterns) are answered in the following Table 5.1 for $\beta>0$ and Table 5.2 for $\beta<0$. In the tables, "T" denotes true, " $F$ " false, " $U$ " unknown so far, and "TT" trivially true. "TF", "TU", "FU", and "TFU" denotes true or false, true or unknown, false or unknown, and true or false or unknown, respectively, depending on what values of boundary data are prescribed or on different parameters in the same region. "Ex" means that there are examples following the proof of the theorem.

Proof. The proof of the theorem follows from verifying conditions $\left(H_{1}^{\prime}\right)$ and $\left(H_{2}^{\prime}\right)$ for $Q_{1}$ and $Q_{2}$, respectively. Though $\left(H_{2}^{\prime}\right)$ and $Q_{2}$ are discussed in the context of stationary solutions, it is more convenient to manipulate the following boundary reconstruction in terms of the output patterns which correspond to the stationary solutions. In the following discussions, $y$ is a global pattern of $(\mathrm{CNN})_{\infty}$ and $\pi_{k}(y)$ is the projection of $y$ onto $T_{k}$. Recall that the output (pattern) for a mosaic pattern at a site $(i, j)$ is 1 ("+") or -1 ("-"). For each $\pi_{k}(y)$, if a reconstruction scheme for the outputs of $\pi_{k}(y)$ near the boundary sites can be obtained so that the reconstructed pattern satisfies the boundary condition, then $\left(H_{1}^{\prime}\right)$ and $\left(H_{2}^{\prime}\right)$ are fulfilled. 


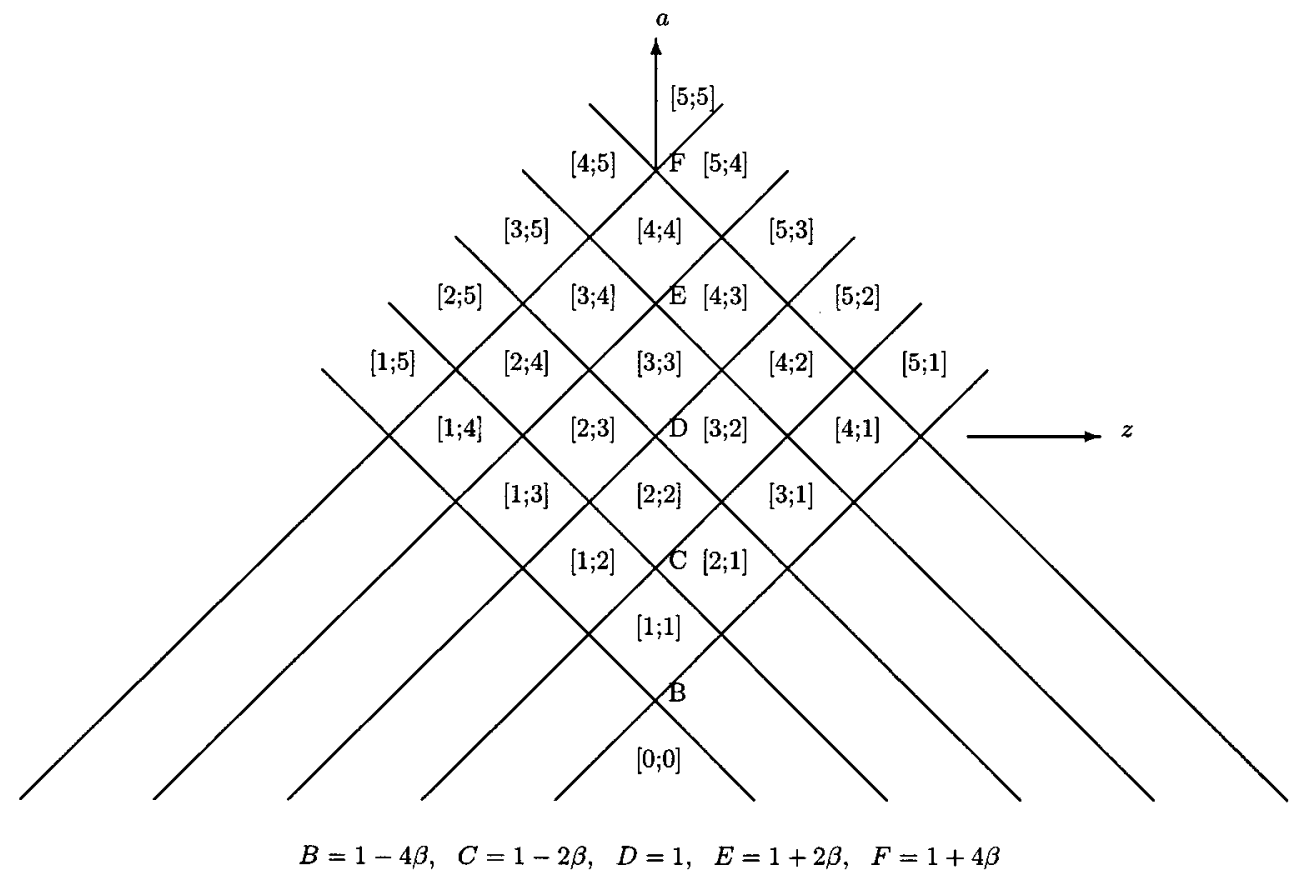

FIG. 5.1. Partition of $z$-a plane, $\beta>0$.

TABLE 5.1

$\beta>0$.

\begin{tabular}{|c|c|c|c|c|c|}
\hline \multicolumn{2}{|c|}{ Boundary condition } & Neumann & Periodic & Dirichlet $D_{1}$ & Dirichlet $D_{0}$ \\
\hline \multirow{2}{*}{$\begin{array}{c}\min \{n]_{\beta} \\
\max \{m, n\} \geq 3\} \geq 4\end{array}$} & $Q_{1}$ & $\mathrm{~T}$ & $\mathrm{~T}$ & $\mathrm{~T}$ & $\mathrm{~T}$ \\
\cline { 2 - 5 } & $Q_{2}$ & $\mathrm{~T}$ & $\mathrm{~T}$ & $\mathrm{~T}$ & $\mathrm{~T}$ \\
\hline$[5 ; 2],[2 ; 5]$ & $Q_{1}$ & $\mathrm{~T}$ & $\mathrm{~T}$ & $\mathrm{TFU}$ & $\mathrm{TU}$ \\
\cline { 2 - 6 }$[4 ; 2],[2 ; 4]$ & $Q_{2}$ & $\mathrm{~T}$ & $\mathrm{~T}$ & $\mathrm{TFU}$ & $\mathrm{TU}$ \\
\hline$[3 ; 3]$ & $Q_{1}$ & $\mathrm{~T}$ & $\mathrm{U}$ & $\mathrm{U}$ & $\mathrm{TU}$ \\
\cline { 2 - 6 } & $Q_{2}$ & $\mathrm{~T}$ & $\mathrm{U}$ & $\mathrm{U}$ & $\mathrm{TF}$ \\
\hline$[3 ; 2],[2 ; 3]$ & $Q_{1}$ & $\mathrm{~T}$ & $\mathrm{~T}$ & $\mathrm{FU}, \mathrm{Ex}$ & $\mathrm{FU}, \mathrm{Ex}$ \\
\cline { 2 - 6 } & $Q_{2}$ & $\mathrm{~T}$ & $\mathrm{~T}$ & $\mathrm{~F}, \mathrm{Ex}$ & $\mathrm{F}, \mathrm{Ex}$ \\
\hline $\min \{m, n\}=1,[2 ; 2]$ & $Q_{1}$ & $\mathrm{TT}$ & $\mathrm{TT}$ & $\mathrm{TT}$ & $\mathrm{TT}$ \\
\hline$[2 ; 2]$ & $Q_{2}$ & $\mathrm{~T}$ & $\mathrm{~T}$ & $\mathrm{~F}$ & $\mathrm{TF}$ \\
\hline
\end{tabular}

$Q_{1}, Q_{2}$ are thus true via Propositions 2.1 and 2.2. In contrast to 1- $d$ lattice in section 4 , in general, we cannot conclude that $Q_{1}, Q_{2}$ are false if an appropriate boundary reconstruction scheme is not found. For some parameters, there are certain $\pi_{k}(y)$ such that as the outputs near the boundary sites are changed, the outputs at more interior sites are forced to change to retain the feasibility. Our answer to $Q_{2}$ is false under such a circumstance. Since the number of such patterns is too complicated to estimate, we may not be able to answer $Q_{1}$ in these cases. Notably, if $h\left(\mathcal{M}^{\infty}\right)=0$, then $h\left(\mathcal{M}_{k}^{B}\right)=0$. This follows from the discussion at the end of section 3. We answer TT (trivially true) to $Q_{1}$ in these cases. 


\begin{tabular}{|c|c|c|c|c|c|}
\hline \multicolumn{2}{|c|}{ Boundary condition } & Neumann & Periodic & Dirichlet $D_{1}$ & Dirichlet $D_{0}$ \\
\hline$[m ; n]_{\beta}$ & & & & & \\
\hline $\min \{m, n\} \geq 4$ & $Q_{1}$ & $\mathrm{~T}$ & $\mathrm{~T}$ & $\mathrm{~T}$ & $\mathrm{~T}$ \\
\cline { 2 - 6 } or $\max \{m, n\}=5$ & $Q_{2}$ & $\mathrm{~T}$ & $\mathrm{~T}$ & $\mathrm{~T}$ & $\mathrm{~T}$ \\
\hline$[4 ; 3],[3 ; 4]$ & $Q_{1}$ & $\mathrm{~T}$ & $\mathrm{U}$ & $\mathrm{T}$ & $\mathrm{T}$ \\
\cline { 2 - 6 }$[4 ; 2],[2 ; 4]$ & $Q_{2}$ & $\mathrm{~T}$ & $\mathrm{U}$ & $\mathrm{T}$ & $\mathrm{T}$ \\
\hline$[3 ; 3]$ & $Q_{1}$ & $\mathrm{U}$ & $\mathrm{U}$ & $\mathrm{U}$ & $\mathrm{TU}$ \\
\cline { 2 - 6 } & $Q_{2}$ & $\mathrm{~F}$ & $\mathrm{~F}$ & $\mathrm{~F}$ & $\mathrm{TF}$ \\
\hline$[3 ; 2],[2 ; 3]$ & $Q_{1}$ & $\mathrm{U}$ & $\mathrm{U}$ & $\mathrm{U}$ & $\mathrm{U}$ \\
\cline { 2 - 6 } & $Q_{2}$ & $\mathrm{~F}$ & $\mathrm{U}$ & $\mathrm{FU}$ & $\mathrm{F}$ \\
\hline$m+n \leq 4$ & $Q_{1}$ & $\mathrm{TT}$ & $\mathrm{TT}$ & $\mathrm{TT}$ & $\mathrm{TT}$ \\
\hline$[2 ; 2]$ & $Q_{2}$ & $\mathrm{~F}$ & $\mathrm{~F}$ & $\mathrm{~F}$ & $\mathrm{~F}$ \\
\hline
\end{tabular}

Let us call the following sites the $l$ th layer of $T_{k},\left\{(l, j),(-l, j),(i, l),(i,-l) \in T_{k}\right.$ : $-l \leq i, j \leq l\}$. In the following, we describe some of these reconstruction schemes. Since the sites at $\{(k, j):-k \leq j \leq k\}$ (respectively, $\{(j, k):-k \leq j \leq k\}$ ) and at $\{(-k, j):-k \leq j \leq k\}$ (respectively, $\{(j,-k):-k \leq j \leq k\}$ ), are far apart if $k$ is large, the only scheme we can derive for periodic B.C. is to amend the $k$ th or even $(k-1)$ th, $(k-2)$ th layers so that the outputs at these sites are all "+" or all "-". This ensures that the first and the last rows (respectively, columns) of $T_{k}$ coincide.

(I) $\beta>0$.

(i) For $\beta>0$, a "+" (respectively, "-") likes to have positive (respectively, negative) states in its neighbors. From our formulation of mosaic patterns and Lemma $5.2, \pi_{k}(y)$ satisfies Neumann B.C. for every global mosaic pattern $y$. Thus, $Q_{1}, Q_{2}$ are true for $(\mathrm{CNN})_{k}-N$ with any $z, a$.

(ii) $(z, a) \in[4 ; 3]$. In the following, we reconstruct the outputs at the $l$ th layers of $T_{k}, l=k-2, k-1, k$. If a "+" in the $(k-2)$ th layer is surrounded by three "-" on $T_{k-2}$ or if a "+" at the corner site is surrounded by two "-" on $T_{k-2}$, we change this "+" to "-". Such a change does not affect the feasibility of its neighboring outputs and the other outputs on $T_{k-2}$. After this step, every "+" on $T_{k-2}$ is adjacent to at least one "+". Next, we set the outputs in the $(k-1)$ th and $k$ th layers to be all "-". It follows that each corner cell on $T_{k}$ is adjacent to two "-" and every other "-" in $T_{k}$ is adjacent to at least three "-". This constructed pattern is a feasible pattern for $(\mathrm{CNN})_{k}-P,(\mathrm{CNN})_{k}-D_{1}$, and $(\mathrm{CNN})_{k}-D_{0}$ with any prescibed boundary data. Figure 5.2 illustrates this scheme. Thus, $Q_{1}, Q_{2}$ are all true.

(iii) $(z, a) \in[4 ; 2]$. The same reconstruction scheme as in $[4 ; 3]$ works as well for $(\mathrm{CNN})_{k}-P$, and $(\mathrm{CNN})_{k}-D_{0}$ (with zero prescribed data). For other boundary data of type $D_{0}$, it is true for some parameters, unknown for some other parameters in this parameter region. If we impose all negative saturated boundary data then, obviously, a reconstructed pattern such as the one in Figure 5.2 satifies $(\mathrm{CNN})_{k}-D_{1}$. If all positive saturated boundary data are imposed, then there is only one feasible pattern satisfying $(\mathrm{CNN})_{k}-D_{1}$. That is, the one with output "+" at each cell. This situation resembles Example 1. For other boundary values, we do not know whether if there exists such a reconstruction scheme. Therefore, $Q_{1}, Q_{2}$ are true for $(\mathrm{CNN})_{k}-P$, true or unknown for $(\mathrm{CNN})_{k}-D_{0}$, and true or false or unknown for $(\mathrm{CNN})_{k}-D_{1}$.

(iv) $(z, a) \in[3 ; 3]$. For $(\mathrm{CNN})_{k}-D_{0}$, we change the outputs in the $k$ th layer so 
that the output at each site on it is identical to the one at the corresponding nearest neighbor in the $(k-1)$-layer, that is, so that $\left(\pi_{k}(y)\right)_{-k, j}=\left(\pi_{k}(y)\right)_{-k+1, j},\left(\pi_{k}(y)\right)_{k, j}=$ $\left(\pi_{k}(y)\right)_{k-1, j},\left(\pi_{k}(y)\right)_{j,-k}=\left(\pi_{k}(y)\right)_{j,-k+1},\left(\pi_{k}(y)\right)_{j, k}=\left(\pi_{k}(y)\right)_{j, k-1}$. With this step, every "+" is adjacent to at least one "+" and every "-" is adjacent to at least one "-". If $(z, a)$ further satisfies $1+\beta<z+a<1+2 \beta, 1+\beta<a-z<1+2 \beta$, which is a subregion of [3; 3], then every $\pi_{k}(y)$ can be reconstructed in its $k$ th layer so that it becomes feasible for $(\mathrm{CNN})_{k}-D_{0}$ (see Figure 5.3). $Q_{1}$ is true in this case. If $(z, a)$ is not in the above-mentioned subregion, some patterns can be found which do not satisfy boundary condition $D_{0}$ and cannot be remedied. Therefore, our answer to $Q_{2}$ is true or false and is true or unknown to $Q_{1}$ for $(\mathrm{CNN})_{k}-D_{0}$. Notably, the answers for $(\mathrm{CNN})_{k}-P$, and $(\mathrm{CNN})_{k}-D_{1}$ are unknown.

(v) $(z, a) \in[3 ; 2]$. For certain prescribed boundary values, only one pattern is feasible for $(\mathrm{CNN})_{k}-D_{1}$ (see Example 1). $Q_{1}$ is false in these cases. In addition, $Q_{2}$ is false for $(\mathrm{CNN})_{k}-D_{1}$ and $(\mathrm{CNN})_{k}-D_{0}$, since for any boundary values of type $D_{1}$ or $D_{0}$, we can find some pattern $\pi_{k}(y)$ which does not satisfy these boundary conditions. If there is a "-" (respectively, "+") in the boundary data, the pattern in Figure 5.4(a) (respectively, (b)), which is the projection $\pi_{k}(y)$ of a global pattern $y$, cannot be a feasible pattern on $T_{k}$ and cannot be amended. Due to some pattern forming properties for parameters in this region, for each $\pi_{k}(y)$, the outputs can be adjusted in the $k$-, $(k-1)$ - and $(k-2)$-layers, so that it becomes a feasible pattern for $(\mathrm{CNN})_{k}-P$.

We omit the cases for the other $[m ; n]$.

(II) $\beta<0$.

(i) $(z, a) \in[5 ; 1]$. For any pattern $\pi_{k}(y)$, we remedy the outputs on the $k$ th layer so that they are all "+". This pattern is feasible for $(\mathrm{CNN})_{k}-N,(\mathrm{CNN})_{k}-P$, $(\mathrm{CNN})_{k}-D_{1}$, and $(\mathrm{CNN})_{k}-D_{0}$ and thus $Q_{1}, Q_{2}$ are true.

(ii) $(z, a) \in[4 ; 4]$. Retain the patterns on $T_{k-2}$. In the $(k-2)$ th layer, if there is a "+" surrounded by three "+" or a "+" at the corner surrounded by two "+", change this "+" to "-". Set the $(k-1)$ th layer to be all "+", the $k$ th layer to be all "-" and the corners of the $k$ th layer (as of $T_{k}$ ) to be all "+". This scheme is effective for all these boundary conditions.

(iii) $(z, a) \in[4 ; 2]$. Given any $\pi_{k}(y)$, retain the pattern on $T_{k-2}$. The first step of the proposed scheme is to change the pattern at each of the four corners of $T_{k-1}$ to "+". Second, if a "+" in the $(k-1)$ th layer is surrounded by three "+" on $T_{k-1}$, we change this "+" to "-". Remedy the $k$ th layer so that output at each site in the $k$ th layer has opposite sign to the output at its nearest neighboring site on the $(k-1)$ th layer. Then, we change some of the "-" outputs in the $k$ th layer again so that there are no two consecutive "-" and the output at each of the four corners in the $k$ th layer is a "+", bordered by two "-", as shown in Figure 5.5. The ones circled there are the outputs to be remedied at each step. Thus, each "+" is surrounded by at least one "-", and each "-" is surrounded by at least three "+". This constructed pattern is feasible for $(\mathrm{CNN})_{k}-N,(\mathrm{CNN})_{k}-D_{1}$, and $(\mathrm{CNN})_{k}-D_{0}$ so that $Q_{1}, Q_{2}$ are true.

(iv) $(z, a) \in[3 ; 3]$. Some patterns $\pi_{k}(y)$ can be found which do not and cannot be modified to satisfy Neumann (see Figure 5.6(a)), periodic (see Figure 5.6(b)), and $D_{1}$ boundary conditions. Thus, $Q_{2}$ is false for these boundary conditions. [3;3] can be further partitioned so that $Q_{2}$ is true for $(\mathrm{CNN})_{k}-D_{0}$ for some $(z, a)$, and false for some other $(z, a)$. This is similar to the discussion in $[3 ; 3], \beta>0$.

Schemes for the other cases resemble the ones presented and so are omitted. The boundary reconstruction schemes we have found in the preceding descriptions involve 


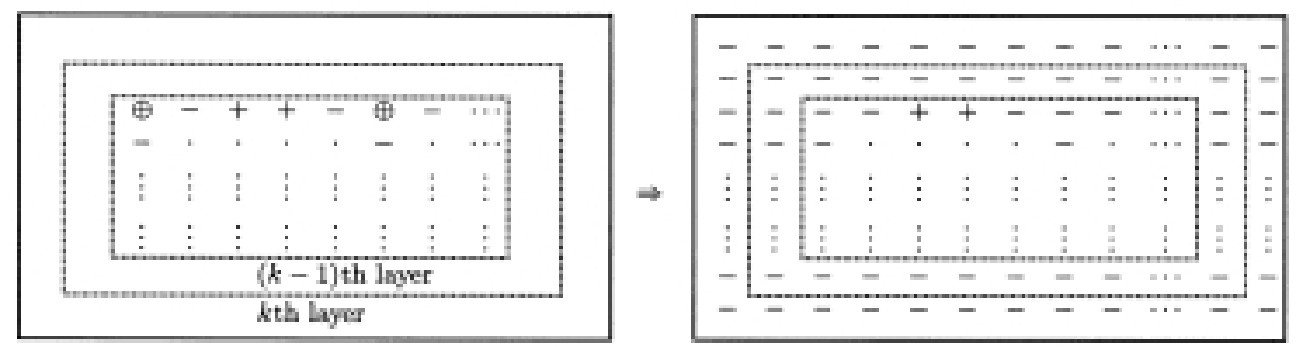

FIG. 5.2. $\beta>0,(z, a) \in[4 ; 3]$.

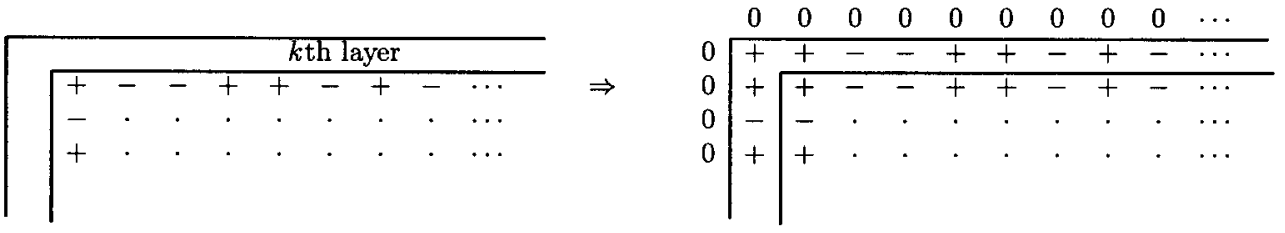

FIG. 5.3. $\beta>0,(z, a) \in[3 ; 3]$.

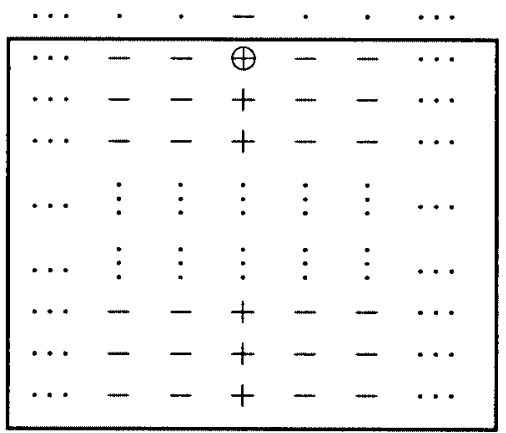

(a)

\begin{tabular}{|ccccccc}
$\cdots$ & $\cdot$ & $\cdot$ & + & $\cdot$ & $\cdot$ & $\cdots$ \\
$\cdots$ & + & - & $\ominus$ & + & - & $\cdots$ \\
$\cdots$ & + & - & - & + & - & $\cdots$ \\
$\cdots$ & + & - & - & + & - & $\cdots$ \\
$\cdots$ & $\vdots$ & $\vdots$ & $\vdots$ & $\vdots$ & $\vdots$ & $\cdots$ \\
$\cdots$ & + & - & - & + & - & $\cdots$ \\
$\cdots$ & + & - & - & + & - & $\cdots$ \\
$\cdots$ & + & - & - & + & - & $\cdots$
\end{tabular}

(b)

FIG. 5.4. $\beta>0,(z, a) \in[3 ; 2]$.

modifying up to three outest layers of $T_{k}$. We believe that, for some parameters in CNN, there exists boundary reconstruction scheme involving more layers so that $Q_{2}$ is true ( $H_{2}^{\prime}$ holds) and $Q_{1}$ is false. Such a scheme is expected to be very complicated.

For the parameters in the region $[5 ; 5]$ with any $\beta$, there is no restriction for the states (patterns) in the nearest neighbors of a "+' or a "-". Such a case is similar to the $[3 ; 3]$ case in a $1-d$ CNN. Thus, if $(z, a) \in[5 ; 5], \pi_{k}(x)$ is feasible on $T_{k}$ with boundary condition $B=N, P, D_{1}, D_{0}$ for every mosaic solution $x$ of $(\mathrm{CNN})_{\infty}$. The setting of the periodic boundary condition here is adjusted to the following: consider $u=\left\{u_{i, j}\right\}_{(i, j) \in T_{k}}, u_{i, j} \in \mathbf{R}$; form a feasible pattern on a $(2 k+1) \times(2 k+1)$ torus by attaching (without overlapping) the first row (respectively, column) to the last row (respectively, column) of $u$, as explained preceding Theorem 4.5. Moreover, for any $(z, a)$ and any $\beta>0, \pi_{k}(x)$ is feasible for $(\mathrm{CNN})_{k}-N$. Thereafter, we have the following results for the phase space $X=\ell^{\infty}$.

Theorem 5.4. Assume that $X=\ell^{\infty}$. 

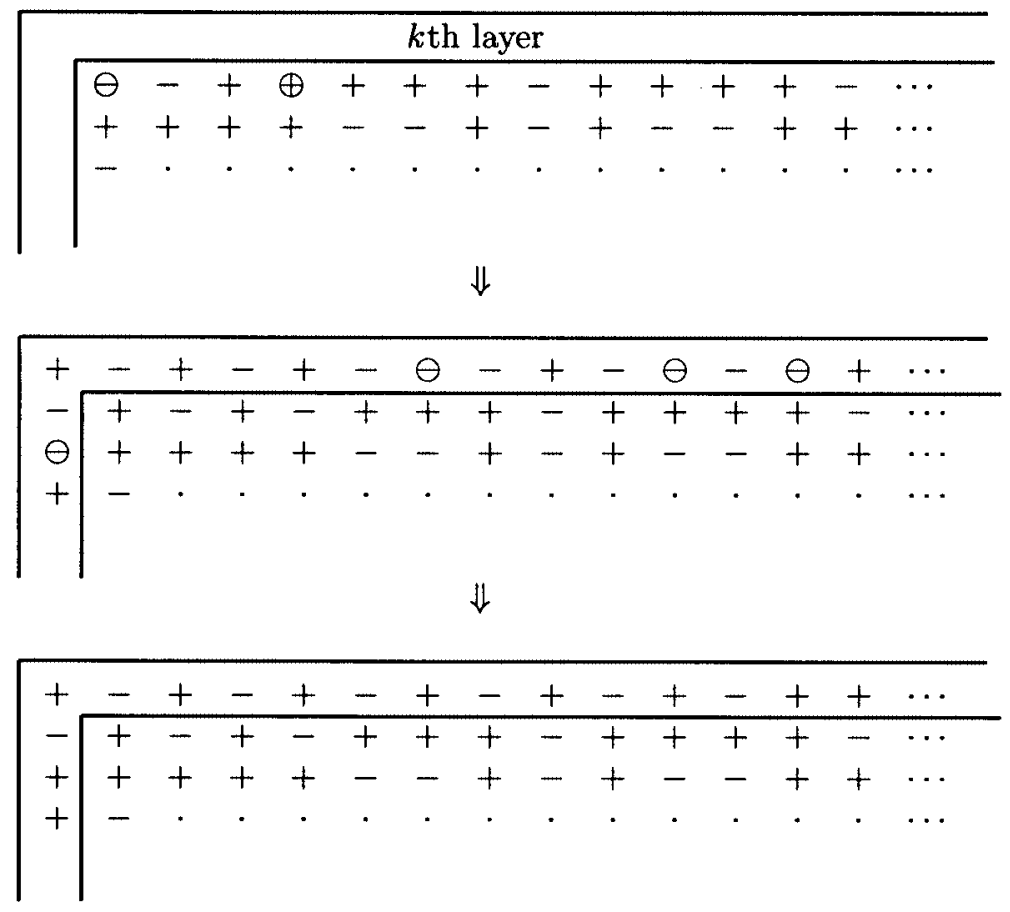

FIG. 5.5. $\beta<0,(z, a) \in[4 ; 2]$.

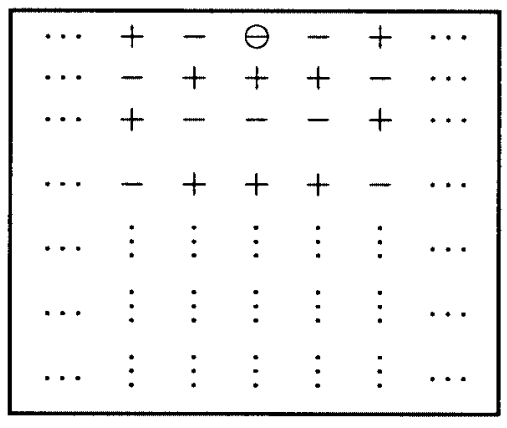

(a)

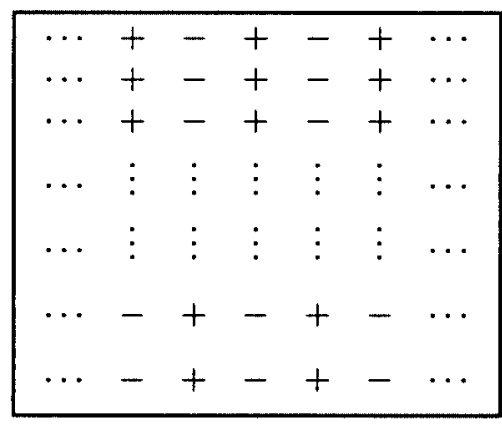

(b)

FIG. 5.6. $\beta<0,(z, a) \in[3 ; 3]$.
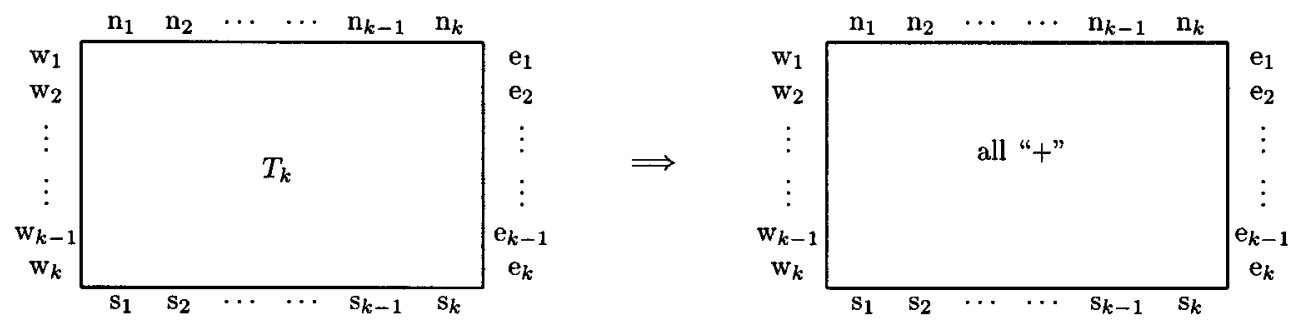
$[3 ; 2]$.

FIG. 5.7. Formation of patterns on $T_{k}$ under Dirichlet B.C. with SA-template, $\beta>0,(z, a) \in$ 


\begin{tabular}{|c|c|c|c|c|c|c|c|c|c|}
\hline+ & & + & - & + & + & $t$ & $t$ & - & $\cdots$ \\
\hline . & $\cdot$ & . & . & . & . & . & . & . & $\cdots$ \\
\hline$\vdots$ & $\vdots$ & : & : & : & : & : & 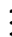 & : & : \\
\hline . & . & . & - & - & . & • & . & . & $\ldots$ \\
\hline . & . & . & • & . & . & . & . & • & $\cdots$ \\
\hline
\end{tabular}

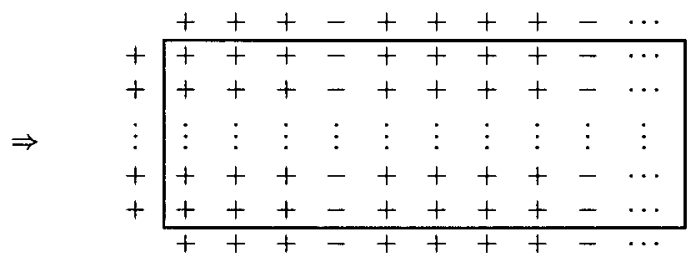
$[4 ; 2]$.

FIG. 5.8. Formation of patterns on $T_{k}$ under Neumann B.C. with HA-template, $\beta<0,(z, a) \in$

(i) Let $\mathcal{U}=\mathcal{M}^{\infty}\left([5 ; 5]_{\beta}\right), \beta \neq 0$, (the set of global mosaic patterns with $(z, a) \in$ $\left.[5 ; 5]_{\beta}\right)$. Then $Q_{2}$ is true for $(\mathrm{CNN})_{k}-B, B=N, P, D_{1}, D_{0}$.

(ii) Let $\mathcal{U}=\mathcal{M}^{\infty}\left([m ; n]_{\beta}\right), \beta>0, m, n \in I[1,5]$. Then $Q_{2}$ is true for $(\mathrm{CNN})_{k}-N$.

Example $1 . h \neq h_{D}$.

Consider $S A$-template with $\beta>0$. In the region [3;2], some prescribed boundary data can be imposed so that there is only one feasible mosaic pattern on $T_{k}$ (Figure 5.7). These data are given as follows:

(i) $\mathrm{e}_{j}, \mathrm{w}_{j}, \mathrm{n}_{j}, \mathrm{~s}_{j}>1$ for all $j \in I[1, k]$.

(ii) $\mathrm{e}_{j}, \mathrm{~s}_{j}>1, \mathrm{w}_{j}, \mathrm{n}_{j}<-1$ for all $j \in I[1, k]$. other $j$.

(iii) $k \geq 3, \mathrm{e}_{j_{1}}, \mathrm{w}_{j_{2}}, \mathrm{n}_{j_{3}}, \mathrm{~s}_{j_{4}}<-1$ for some $j_{l} \in I[1, k]$ and $\mathrm{e}_{j}, \mathrm{w}_{j}, \mathrm{n}_{j}, \mathrm{~s}_{j}>1$ for the

(iv) Some boundary states with values of magnitude less than one can also be imposed to yield the same result. Notably,

$$
(z, a) \in[3 ; 2] \Leftrightarrow 0<z-a+1<2 \beta \text { and } 0<z+a-1<2 \beta .
$$

If we consider $(z, a)$ in the subregion of [3;2], which satisfies $\tau \beta<z-a+1<2 \beta$ for some $0<\tau<2$, then the total output of a negative saturated state $T O(-)$ is less than $-\tau$, since $T O(-)$ satisfies

$$
x_{i, j}=z-a+\beta \cdot T O(-)<-1 .
$$

If we set $\mathrm{e}_{j}, \mathrm{w}_{j}, \mathrm{n}_{j}, \mathrm{~s}_{j} \geq 2-\tau$ for each $j \in I[1, k]$, then the circled "-" in (5.5), which is at the upper-left corner of $T_{k}$, has total output $T O(-) \geq-\tau$ and yields a contradiction:

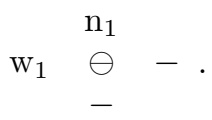

Consequently, the cell at the upper-left corner of $T_{k}$ must have the output pattern "+". In addition, every other cell on $T_{k}$ must also have the output pattern "+". The picture in Figure 5.7 remains true.

Example 2. $h \neq h_{N}$.

Consider $H A$-template. The set of tentative basic mosaic patterns $\mathcal{B}([m ; n])$ corresponding to each parameter region $[m ; n], m, n \in I[0,5]$ can be described as follows. Recall the notations in (5.4). If $(z, a) \in[m ; n], \beta>0$ (respectively, $\beta<0$ ), then the corresponding tentative basic mosaic patterns with "+" at the center, that is, of the form in (5.4a), are those with at least $(5-m)$ " + " (respectively, "-") in $\{\mathrm{e}, \mathrm{w},-\mathrm{s}$, $\mathrm{n}\}$; the corresponding tentative basic mosaic patterns with " -" at the center, that is, of the form in $(5.4 \mathrm{~b})$, are those with at least $(5-n)$ "-" (respectively, "+") in 
$\{\mathrm{e}, \mathrm{w},-\mathrm{s}, \mathrm{n}\}$. Herein, $-\mathrm{s}=$ "+" (respectively, "-") if s ="-" (respectively, "+"). According to a previous investigation [37], CNN with $H A$-template exhibits spatial chaos if and only if $\min \{m, n\} \geq 3$ with $\beta>0$ and $\min \{m, n\} \geq 2, m+n \geq 6$ with $\beta<0$. In fact, $h\left(\mathcal{M}^{\infty}([4 ; 2])\right)>(\ln 2) / 16$ if $\beta<0$. In this example, we would like to show that $h\left(\mathcal{M}^{\infty}([4 ; 2])\right)>h_{N}\left(\mathcal{M}^{N}([4 ; 2])\right)=0$ if $\beta<0$. Consider $\beta<0$ and $(z, a) \in[4 ; 2]$. There is a character for the pattern forming in this parameter region: if $y_{i, \ell}=-1$ and $y_{i, \ell+1}=-1$, then it yields $y_{i-1, \ell}=1, y_{i+1, \ell}=1, y_{i, \ell-1}=-1$, and then $y_{i-1, j}=1, y_{i+1, j}=1, y_{i, j-1}=-1$ for all $j \leq \ell$. The formation of patterns on $T_{k}$ under Neumann B.C. can thus be described as follows. On the top of the $k$ th layer, if there is a horizontal string of $\ell$ " + ", on the sites $(i, k), i \in[p, p+\ell-1]$, bordered by "-" at two ends if $-k<p<k$ or by "-" at one end if $p=-k$ or $k$, then it must be all "+" below each of these "+", and it must be all "-" below each of these "-", as illustrated in Figure 5.8. Thus, if Neumann B.C. is imposed, the number of patterns on $T_{k}$ is less than or equal to $2^{k}$; therefore, $h_{N}\left(\mathcal{M}^{N}([4 ; 2])\right)=0$.

Acknowledgments. The author has benefited from several fruitful discussions with Jonq Juang, Song-Sun Lin, and Wen-Wei Lin. He learned this very interesting open problem $Q_{1}$ from V. S. Afraimovich. Comments on the related studies in PDE from Jack Hale were very inspiring. The suggestions and comments from the referees led to several improvements of this work. All these are gratefully acknowledged.

\section{REFERENCES}

[1] R. Artuso, P. Cvitanovic and G. Casati, eds., Chaos, Order and Patterns, Plenum Press, New York, 1990.

[2] V. S. Afraimovich and S. B. Hsu, Lectures on Chaotic Dynamical Systems, National TsingHua Univ., Taiwan, 1997.

[3] V. S. Afraimovich and L. A. Bunimovich, Density of defects and spatial entropy in extended systems, Phys. D, 80 (1995), pp. 277-288.

[4] V. S. Afraimovich And V. I. Nekorkin, Chaos of traveling waves in a discrete chain of diffusively coupled maps, Internat. J. Bifur. Chaos, 4 (1994), pp. 631-637.

[5] V. S. Afraimovich And S.-N. Chow, Existence of evolution operators group for infinite lattice of coupled ordinary differential equation, Dynam. Systems Appl., 3 (1994), pp. 155-174.

[6] J. BELL, Some threshold results for modes of myelinated nerves, Math. Biosci., 54 (1981), pp. 181-190.

[7] J. W. CAHN, Theory of crystal growth and interface motion in crystalline materials, Acta Metallurgica, 8 (1960), pp. 554-562.

[8] J. W. CAhn, S.-N. Chow, AND E. S. VAn Vleck, Spatially discrete nonlinear diffusion equations, Rocky Mountain J. Math., 25 (1995), pp. 87-118.

[9] S.-N. Chow, J. Mallet-Paret, and E. S. Van Vleck, Pattern formation and spatial chaos in spatially discrete evolution equations, Random Comput. Dynam., 4 (1996), pp. 109-178.

[10] S.-N. Chow And J. Mallet-Paret, Pattern formation and spatial chaos in lattice dynamical system I, IEEE Trans. Circuits Systems, 42 (1995), pp. 746-751.

[11] S.-N. CHOw AND W. Shen, Stability and bifurcation of traveling wave solutions in coupled map lattices, Dynam. Systems Appl., 4 (1995), pp. 1-26.

[12] S.-N. Chow, J. Mallet-Paret, and E. S. Van Vleck, Dynamics of lattice differential equations, Internat. J. Bifur. Chaos, 6 (1996), pp. 1605-1622.

[13] L. O. ChuA And L. YAng, Cellular neural networks: Theory, IEEE Trans. Circuits Syst., 35 (1988), pp. 1257-1272.

[14] L. O. Chua And L. Yang, Cellular neural networks: Applications, IEEE Trans. Circuits Syst., 35 (1988), pp. 1273-1290.

[15] L. O. Chua And T. Roska, The CNN paradigm, IEEE Trans. Circuits Systems, 40 (1993), pp. $147-156$.

[16] L. O. ChuA, CNN: A vision of complexity, Internat. J. Bifur. Chaos, 7 (1997), pp. 2219-2425.

[17] L. O. ChuA AND B. Shi, Multiple layer cellular neural networks-a tutorial, in Algorithms and Parallel VLSI Architectures, vol. A, A. J. Van der Veen and E. F. Deprette, eds., North-Holland, Amsterdam, 1991, pp. 137-168. 
[18] H. E. Cook, D. DE Fontaine, and J. E. Hilliard, A model for diffusion on cubic lattices and its application to the early stages of ordering, Acta Metallurgica, 17 (1969), pp. 765-773.

[19] P. Coullet And P. Huerre, Eds., New Trends in Nonlinear Dynamics and Pattern-Forming Phenomena: The Geometry of Nonequilibrium, Plenum Press, New York, 1990.

[20] K. R. Crounse, P. Thiran, G. Sett, and L. O. Chua, Characterization and dynamics of pattern formation in cellular neural network, Internat. J. Bifurc. Chaos, 6 (1997), pp. 17031724.

[21] R. Dillon, P. K. Maini, and H. G. Othmer, Pattern formation in generalized Turing systems, I. Steady-state patterns in systems with mixed boundary conditions, J. Math. Biol., 32 (1994), pp. 345-393.

[22] T. ERneux And G. Nicolis, Propagating waves in discrete bistable reaction-diffusion systems, Phys. D, 67 (1993), pp. 237-244.

[23] B. Fiedler, Do global attractors depend on boundary conditions?, Doc. Math., 1 (1996), pp. 215-228.

[24] W. J. Firth, Optical memory and spatial chaos, Phys. Rev. Lett., 61 (1988), pp. 329-332.

[25] R. A. Gardner, Global continuation of branches of nondegenerate solutions, J. Differential Equations, 61 (1986) pp. 321-334.

[26] J. Hale and C. Rocha, Varying boundary conditions with large diffusivity, J. Math. Pures Appl., 66 (1987), pp. 139-158.

[27] J. Hale, Asymptotic Behavior of Dissipative Systems, AMS, Providence, RI, 1988.

[28] E. J. Holder and D. G. Schaeffer, Boundary condition and mode jumping in the von Kármán equations, SIAM J. Math. Anal., 15 (1984) pp. 446-458.

[29] J. JuAng AND S. S. Lin, Cellular neural networks I: mosaic pattern and spatial chaos, SIAM J. Appl. Math., 60 (2000), pp. 891-915.

[30] J. P. KeEner, Propagation and its failure in coupled systems of discrete excitable cells, SIAM J. Appl. Math., 47 (1987), pp. 556-572.

[31] J. P. Laplante And T. Eveneux, Propagation failure in arrays of coupled bistakle chemical reactors, J. Phys. Chem., 96 (1992), pp. 4931-4934.

[32] S. S. Lin AND C. W. Shin, Complete stability for standard cellular neural networks, Internat. J. Bifur. Chaos, 9 (1999), pp. 909-918.

[33] Z. Mei AND F. Theil, Variation of bifurcations along a homotopy from Neumann to Dirichlet problems, Nonlinear Anal., 27 (1996), pp. 1381-1395.

[34] A. Mielke, The complex Ginzburg-Landau equation on large and unbounded domains: Sharper bounds and attractors, Nonlinearity, 10 (1997), pp. 199-222.

[35] C. Robinson, Dynamical Systems, CRC Press, Boca Raton, FL, 1995.

[36] D. Schaeffer and M. Golubitsky, Boundary conditions and mode jumping in the buckling of a rectangular plate, Comm. Math. Phys., 69 (1979) pp. 209-236.

[37] C. W. SHiH, Pattern formation and spatial chaos for cellular neural network with asymmetric templates, Internat. J. Bifur. Chaos, 8 (1998), pp. 1907-1936.

[38] P. Thiran, K. R. Crounse, L. O. Chua, and M. Hasler, Pattern formation properties of autonomous cellular neural networks, IEEE Trans. Circuits Syst., 42 (1995), pp. 757-774.

[39] P. ThIRAN, Influence of boundary conditions on the behavior of cellular neural networks, IEEE Trans. Circuits Syst. Fund. Theory Appl., 40 (1993), pp. 207-212. 